\title{
Shaping of the Female Human Brain by Sex Hormones: A Review
}

\author{
Elisa Rehbein ${ }^{a}$ Jonas Hornung ${ }^{a}$ Inger Sundström Poromaa b Birgit Derntla, c, d \\ ${ }^{a}$ Department of Psychiatry and Psychotherapy, Innovative Neuroimaging, University of Tübingen, Tübingen,

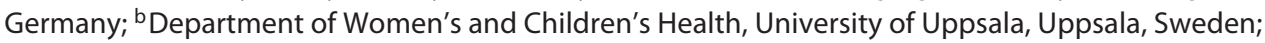 \\ 'Werner Reichardt Centre for Integrative Neuroscience, University of Tübingen, Tübingen, Germany; \\ ${ }^{\mathrm{d}}$ Lead Graduate School, University of Tübingen, Tübingen, Germany
}

\section{Keywords}

Sex hormones · Brain structure · Gray matter

\begin{abstract}
Traditionally sex hormones have been associated with reproductive and developmental processes only. Since the 1950s we know that hormones can have organizational effects on the developing brain and initiate hormonal transition periods such as puberty. However, recent evidence shows that sex hormones additionally structure the brain during important hormonal transition periods across a woman's life including short-term fluctuations during the menstrual cycle. However, a comprehensive review focusing on structural changes during all hormonal transition phases of women is still missing. Therefore, in this review structural changes across hormonal transition periods (i.e., puberty, menstrual cycle, oral contraceptive intake, pregnancy and menopause) were investigated in a structured way and correlations with sex hormones evaluated. Results show an overall reduction in grey matter and region-specific decreases in prefrontal, parietal and middle temporal areas during puberty. Across the menstrual cycle grey matter plasticity in the hippocampus, the amygdala as well as temporal and pa-
\end{abstract}

karger@karger.com www.karger.com/nen

(C) 2020 S. Karger AG, Basel

Karger' rietal regions were most consistently reported. Studies reporting on pre-and post-pregnancy measurements revealed volume reductions in midline structures as well as prefrontal and temporal cortices. During perimenopause, the decline in sex hormones was paralleled with a reduction in hippocampal and parietal cortex volume. Brain volume changes were significantly correlated with estradiol, testosterone and progesterone levels in some studies, but directionality remains inconclusive between studies. These results indicate that sex hormones play an important role in shaping women's brain structure during different transition periods and are not restricted to specific developmental periods.

(c) 2020 S. Karger AG, Basel

\section{Introduction}

During ontogenesis, humans undergo rapid and extensive changes in brain structure and function. Even upon completion of this process, human brains remain flexible and are able to adapt to different environments. Factors contributing to these changes involve (i) behavioural adaptations, for example, through learning and (ii) environmental context-dependent aspects, for example, 
stressful events and hormonal transition periods [1]. Of special interest of this review are sex hormones, which fluctuate considerably during a woman's reproductive lifespan and influence structural changes in the brain. Traditionally, the influence of sex hormones on the brain is discussed in terms of activational and organizational aspects [2]. Since the late 1950s it is known that sex hormones have the capacity to shape the brain during development. Accordingly, a dichotomization of the sexes begins early on during the prenatal period, induced by a surge of testosterone, leading to sexual differentiation of brain tissues and brain development [3].

The brain represents an important target for oestrogen and progesterone effects. Both hormones provide specific neuroendocrine conditions through which brain structure and function are modulated across a woman's life span. The trophic effects of ovarian hormones emerge early in brain development and remain throughout adolescence and adulthood $[4,5]$. The production of gonadal sex hormones during puberty, for example, regulates an extensive reorganization of the brain [6]. Moreover, neural alterations have also been observed in response to even subtle changes in endogenous or exogenous sex hormone levels later in life [7, 8]. Many of these actions occur in brain regions crucial for a variety of cognitive and emotional processes [7, 9]. Furthermore, gonadal hormones impact brain development and plasticity via, for example, neurite outgrowth and synaptogenesis [10], dendritic branching [11], and myelination [12].

Animal studies provide evidence that sex hormones can bind to hormone receptors in the brain leading to slower genomic effects, that is, involving gene transcription, or having faster non-genomic effects, that is, by triggering fast signal cascades [13-16]. In animal studies, a larger density of sex steroid receptors has been reported in a number of brain regions including hippocampus and claustrum for oestrogen [17] and amygdala, cerebellum, hippocampus, and hypothalamus for progesterone [18]. Hormones can also be directly synthesized in the brain, as so-called neurosteroids, and act via rapid membrane receptors [19]. In animal models, the administration of progesterone and its metabolites dihydro-progesterone and tetrahydro-progesterone increased myelination after experimentally induced demyelination [20] and decreased cell death after stroke [21]. Post-mortem analyses pointed to higher hormone concentrations of progesterone in brain regions like the amygdala, the cerebellum and the hypothalamus and of allopregnanolone in the substantia nigra and the basal hypothalamus [22]. In the same post-mortem study, the authors found that brain progesterone concentrations were increased in naturally cycling (NC) women during their luteal cycle phase compared to postmenopausal women. Thus, also in humans, concentrations of available sex steroids in the brain change and may exert an effect on brain structure. Sex hormones and their metabolites can also interact with the brain's neurotransmitter systems by modulating the release of glutamate, GABA, acetylcholine, norepinephrine, dopamine as well as serotonin (for review please see [23]). These biological mechanisms may have far reaching impact on structural brain changes and lead to behavioural consequences. Receptors of sex hormones such as estradiol (ERalpha, ERbeta), progesterone (PRA, PRB) or testosterone (androgen receptors) are highly expressed in areas important for emotional processing, for example, hypothalamus and the limbic system [24, 25]. High ER alpha expression in areas like the hippocampus points toward its role in memory and spatial recognition [26].

The term oestrogen describes a class of structurally and functionally similar compounds, the most common of which are estrone (E1), estradiol (E2) and estriol (E3) [27]. Of these, estradiol is the most potent endogenous oestrogen in humans $[28,29]$. The predominant source for oestrogen production is the antral follicles of the ovary, and by involvement of 2 different cell types, that is, the theca and granulosa cells of the growing follicle, it is synthesized via aromatization of testosterone. Estradiol is present in women and men, although adult men show considerably lower serum levels than women [30], with minimal fluctuations or age-related decline [31,32]. Conversely, non-pregnant premenopausal women exhibit biphasic cyclic variation in serum estradiol during their 28day menstrual cycle, with lowest levels during the early follicular (EF) phase (ca. $150 \mathrm{pmol} / \mathrm{L}$ ), the highest levels around ovulation (OV; around $670 \mathrm{pm} / \mathrm{L})$ and a second, smaller peak during the mid-luteal (ML) phase (around $500 \mathrm{pm} / \mathrm{L}$ ) [30,33]. Inter-individual variation in estradiol synthesis during the menstrual cycle is profound, especially in the preovulatory phase where concentrations of $1,500 \mathrm{pmol} / \mathrm{L}$ or more can be found [34]. During pregnancy, estradiol increases up to 9-fold but drops dramatically post-partum [35]. Similarly, estradiol declines dramatically following menopause, usually with levels beyond $100 \mathrm{pm} / \mathrm{L}$ [36]. Progesterone plays a crucial role in both female and male health and development. In adult males, serum progesterone levels reportedly range between 1 and $3 \mathrm{~nm} / \mathrm{L}$ with little change relative to age [30, 37]. In contrast, non-pregnant premenopausal women experience variable serum progesterone levels relative to their menstrual cycle with lowest concentrations report- 
ed during the EF phase $(0.3-2 \mathrm{~nm} / \mathrm{L})$ and maximum concentrations during the ML phase $(20-55 \mathrm{~nm} / \mathrm{L})[30,38]$. Progesterone levels are even higher during pregnancy, with serum progesterone steadily increasing throughout the gestational period and reaching peak concentrations of up to fourfold non-pregnant levels at term $[35,39]$. As is the case for estradiol, serum progesterone levels dramatically decline following menopause [40].

Across the lifespan, hormonal fluctuations lead to pivotal actions in the human body. Hormonal transition periods such as puberty, pregnancy/postpartum, menopause and even subtler fluctuations during the menstrual cycle seem to predispose women to mood disorders [4143]. While some highlight the impact of estradiol on the synthesis, availability and metabolism of serotonin [44], others stress the role of progesterone as well as allopregnanolone, a GABA-active progesterone metabolite, as causal agents for premenstrual syndrome and premenstrual dysphoric disorder $[45,46]$, potentially via an inverted U-shape relationship between allopregnanolone and psychological well-being [47]. A recent review highlights the role of allopregnanolone for psychiatric symptoms, though evidence is limited and rare for some transition periods [48]. Catenaccio et al. [49] reviewed the association of sex hormones during hormonal transition periods and brain structure in women (including menstrual cycle, pregnancy and menopausal transitions) and found most prominent effects of sex hormones on limbic brain structures with mediating effects of oestrogen and progesterone on emotional processing.

\section{Influence of Sex Hormones across a Woman's Life}

Following early hormonal influences, puberty marks an important hormonal transition period involving the start of the reproductive period, along with several physical, social and emotional changes [50]. Puberty is usually induced by an increase in frequency and amount of the secretion of gonadotropin-releasing hormone $(\mathrm{GnRH})$, which in turn directs and stimulates the synthesis of gonadotropins, the follicle stimulating hormone and the luteinizing hormone $[51,52]$. In recent years kisspeptin, the peptide ligand of the G-coupled protein receptor (GPR54), has shown to be an important gate keeper and initiator of puberty onset by directly stimulating $\mathrm{GnRH}$ production (for review please see $[53,54]$ ). Increases in gonadal hormones, that is, testosterone, estradiol and progesterone lead to the development of secondary sex characteristics with estradiol and progesterone

Shaping of the Female Brain by Sex

Hormones being more prominent in girls and testosterone in boys. Even though estradiol levels are much higher in girls, additional transformation of testosterone to estradiol occurs equally in boys [55]. In general, there exists a natural variation of puberty onset of around $4-5$ years [56]. Due to these individual differences, objective measures of pubertal stage such as Tanner stage [57] or self-rating (pubertal development scale; [58]) are crucial. Sex differences in the onset of puberty are seen with boys usually developing around 1-2 years later than girls [59].

Brain maturation in this period consists of pruning of neuronal connections and increased myelination, which follows a posterior-anterior direction [60]. Accordingly, prefrontal areas may not be fully matured until the early 20s [61]. While white matter increases follow a linear pattern through adolescence, grey matter structural changes follow an inverted U-shape with a peak in late childhood and significant decreases especially in dorsal frontal and parietal areas during adolescence [60, 62]. However, trajectories between specific brain areas differ regarding age onset of maturation and pubertal stage $[60,63]$. Overall, these changes lead to an improvement in cognitive and social abilities [64]. Due to the different trajectories of brain maturation, the interplay of an immature prefrontal cortex and increased activation in subcortical areas lead to difficulties in regulatory processes and risk taking in adolescents [65]. Evidence from several animal studies point towards an important role of sex hormones in these structural changes [52].

Besides drastic hormonal changes in puberty, every woman will undergo monthly fluctuations in hormonal levels, that is, the menstrual cycle. As described previously, the average human menstrual cycle is 28 days long and divided into 4 different phases during which hormonal levels fluctuate in a systematic manner [9]. In the EF phase both estradiol and progesterone levels are low. Estradiol steadily increases during the late follicular (LF) phase until its peak shortly before OV and then declines before reaching another peak during the ML phase. The early luteal phase begins with a rise in both estradiol and progesterone, with a peak in both hormones approximately 1 week after $\mathrm{OV}$, and is followed by a steep decline in the late luteal (LL) phase, leading to menses. During their reproductive year's millions of women around the world use some form of oral contraceptives (OCs) to prevent unwanted pregnancies. These usually contain a combination of ethinylestradiol and synthetic progestins. OC intake thereby reduces endogenous hormones levels of estradiol and progesterone significantly [66].

Neuroendocrinology 2021;111:183-206 
Many women undergo pregnancy at least once in their lives during which women experience the peak of hormonal levels. During the post-partum period this process is reversed again; after a steep decline of hormones, women reach pre-gravid hormone levels in around 5 days [67]. Pregnancy and the transition to the post-partum period involves a multitude of hormones, including prolactin, oxytocin, and the hormones of the hypothalamus-pituitary-adrenal axis.

Menopause marks the last hormonal transition period in the life of a woman. During the perimenopausal period, which corresponds to the 1-2 years preceding the final menstruation, estradiol levels show a high variability but are gradually decreasing in comparison with the premenopausal period. After menopause, estradiol levels continue to decrease, and reach postmenopausal levels after 2-3 years [68]. During or following this decline, women commonly experience a number of vasomotor symptoms like sweating, hot flashes [69] and sleep problems [70]. To counteract the vasomotor symptoms, many women are being prescribed menopausal hormone therapy (MHT) - either oestrogen only (only hysterectomized women) or a combination of oestrogen with progesterone [71] - to restore, to some degree, the loss of pre-menopausal hormone levels. For this review, we were interested in learning whether the intake of MHT had a differential impact on grey matter volume (GMV) compared to untreated women.

To the best of our knowledge a systematic review on structural changes across different hormonal transition periods that also includes the important developmental phase of puberty is still missing. Therefore, the aim of this review is to track the impact of hormonal changes throughout a woman's life on brain structure and volume starting with puberty, followed by cyclic hormonal fluctuations within the menstrual cycle, potential intake of OCs, pregnancy and the cessation or substitution of sex hormones in the peri- and post-menopausal phase.

\section{Methods}

An internet-based search on PubMed was conducted separately for the phases of puberty, menstrual cycle/OC and menopause. Search terms included either (puberty)/(menstrual cycle) OR (oral contraceptives/anti-baby pill) OR (menopause) OR (pregnancy) OR (post-partum period) AND (neuroimaging) OR (MRI) AND (brain structure) AND (human) as well as cited studies in the reference section of the previous selected papers. In total, 461 studies were screened including all hormonal transition periods (puberty [87], menstrual cycle [30], OCs [9], pregnancy [264], postpartum [33], menopause [38]). Exclusion criteria consisted of non-human studies, functional MRI, as well as foetal MRI during pregnancy. In addition, pubertal stage had to be assessed either by the use of Tanner stage or Pubertal development scale. Thirty studies that fulfilled the abovementioned criteria were finally included. Nine studies overlap with the review of Catenaccio et al. [49], which did not include studies during puberty and white matter.

\section{Results}

\section{Puberty}

In total, 10 studies showing associations between sex hormones levels (i.e., estradiol, testosterone and LH) and changes in grey and white matter during puberty were included in this review (for details, please see Table 1). No studies assessing progesterone and brain structure were found.

\section{Global Grey Matter}

Overall, a decrease of grey matter over the course of puberty and with increasing age has been consistently reported in boys and girls $[72,73]$. As a discussion of sex differences in maturation is beyond the scope of this review, the focus will be on maturation in girls only (for reports of sex differences see online suppl. 2, see www. karger.com/doi/10.1159/000507083 for all online suppl. materials).

In a longitudinal study, covering 2 years in an age range of 10-14 years, high estradiol levels predicted increases in the left middle temporal gyrus (MTG) [74]. Contrarily, Peper et al. [75] reported a negative association between overall GMV and estradiol in a cross-sectional study assessing twins and siblings. In addition, specific brain areas including the superior- and inferior prefrontal, orbitofrontal, parietal as well as temporal cortices showed a decrease in grey matter across age and a negative association with estradiol. Koolschijn et al. [76] reported a negative association between estradiol and anterior cingulate (ACC) GMV in a cross-sectional study. Interestingly, the opposite, that is, a positive correlation between estradiol levels and GMV was found in the middle frontal-, inferior temporal- and middle occipital gyri [75]. A positive relationship between estradiol and brain volume was also reported for the uncal cortex and parahippocampus [77].

Negative correlations were reported for testosterone and orbitofrontal cortex volume, thinning of the right medial superior frontal surface area, volume of the right amygdala, hippocampus, parietal cortex (including precuneus and superior parietal cortex) and smaller cortical volumes [76-78]. Conversely, a positive relationship was 
Table 1. Puberty

\begin{tabular}{llll}
\hline Study Groups & Design & $\begin{array}{l}\text { Measurement of } \\
\text { puberty }\end{array}$ & $\begin{array}{l}\text { Results correlations/regression } \\
\text { hormones }\end{array}$ \\
\hline
\end{tabular}

Gray matter

$\begin{array}{llll}\text { Herting et al. [80], } & N=126(63 \text { girls }), & \begin{array}{l}\text { Longitudinal } \\ \text { age range } 10-14 \text { years }\end{array} & \begin{array}{l}\text { Tanner stage (trained } \\ \text { research nurse })\end{array}\end{array}$

EST prediction (time*E2):

age range $10-14$ years $\quad(\sim 2$ years $)$

(-) for low levels: amygdala volume,

(+) for high levels: amygdala;

(+) for low levels: pruning effects grey matter

TEST (time*T interaction):

(+) for low levels: caudate volume,

$(-)$ for high levels: caudate volume

\begin{tabular}{|c|c|c|c|c|}
\hline $\begin{array}{l}\text { Herting et al. [74], } \\
2015^{*}\end{array}$ & $\begin{array}{l}N=126(63 \text { girls }) \\
\text { age range } 10-14 \text { years }\end{array}$ & $\begin{array}{l}\text { Longitudinal } \\
(\sim 2 \text { years })\end{array}$ & $\begin{array}{l}\text { Tanner stage (trained } \\
\text { research nurse) }\end{array}$ & $\begin{array}{l}\text { TEST (-): middle superior frontal } \\
\text { surface area } \\
\text { EST (-): L STG cortical thickness }\end{array}$ \\
\hline $\begin{array}{l}\text { Koolschijn et al. [76], } \\
2014\end{array}$ & $\begin{array}{l}N=215(112 \text { girls }) \\
\text { age range } 8-25 \text { years }\end{array}$ & Cross-sectional & PDS (up to age 18) & $\begin{array}{l}\text { TEST (-): ACC/OFC volumes } \\
\text { EST (-): ACC volume }\end{array}$ \\
\hline $\begin{array}{l}\text { Paus et al. [79], } \\
2010\end{array}$ & $\begin{array}{l}\text { Saguenay Youth } \\
\text { Study } N=419 \\
\text { ( } 215 \text { girls) } \\
\text { age range } \\
12-18 \text { years }\end{array}$ & Cross-sectional & - & $\begin{array}{l}\text { TEST }(+) \text { : brain volume, absolute and } \\
\text { relative WM, WM to GM ratio } \\
\text { TEST (-): relative GM }\end{array}$ \\
\hline $\begin{array}{l}\text { White matter } \\
\text { Herting et al. [81], } \\
2012\end{array}$ & $\begin{array}{l}N=77 \text { (39 girls) } \\
\text { age range } 10-16 \text { years }\end{array}$ & Cross-sectional & - & $\begin{array}{l}\text { EST (-): FA R AG WM } \\
\text { L SLF } \\
\text { TEST(+): R precentral gyrus WM }\end{array}$ \\
\hline $\begin{array}{l}\text { Peper et al. [73], } \\
2008\end{array}$ & $\begin{array}{l}N=104 \\
\text { monozygotic }(22 \text { girls }) / \\
\text { dizygotic twins }(25 \text { girls }) \\
\text { mean age: } 9 \text { years }\end{array}$ & Cross-sectional & $\begin{array}{l}\text { Tanner Stage } \\
\text { questionnaire } \\
\text { administered by } \\
\text { trained researcher } \\
\text { (no self-report) }\end{array}$ & $\begin{array}{l}\text { LH (+): regional WM density: } \\
\text { L Cingulum } \\
\text { L/R MTG } \\
\text { R SFG } \\
\text { splenium of the corpus callosum }\end{array}$ \\
\hline
\end{tabular}

* Same study population. Studies investigating the influence of sex hormones on changes in brain structure during puberty. Only significant results were listed. MNI coordinates can be found in online supplementary 1. (+), positive correlation; (-), negative correlation; STG, superior temporal gyrus; FA, fractional anisotropy; AG, angular gyrus; SLF, superior longitudinal fasciculus; WM, white matter; ACC, anterior cingulate cortex; HIPP, hippocampus; parahipp., parahippocampus; MTG, middle temporal gyrus; OFC, orbitofrontal cortex; GM, grey matter; SMG, supramarginal gyrus; MTG, middle temporal gyrus; IFG, inferior frontal gyrus; MFG, middle frontal gyrus; ITG, inferior temporal gyrus; MOG, middle occipital gyri; PDS, pubertal development scale. 
Table 2. Menstrual cycle/oral contraceptives

\begin{tabular}{|c|c|c|c|c|}
\hline Study & Groups ( $n$, age) & Design & $\begin{array}{l}\text { Results changes in brain structure } \\
\text { (i.e., volume, brainAGE, ADC, } \\
\text { MD) }\end{array}$ & $\begin{array}{l}\text { Results correlations } \\
\text { hormones }\end{array}$ \\
\hline $\begin{array}{l}\text { Gray matter } \\
\text { Franke et al. [95], } \\
2015^{*}\end{array}$ & $\begin{array}{l}N=8 \mathrm{NC} \\
\text { age range 21-31 years }\end{array}$ & $\begin{array}{l}\text { Longitudinal } \\
\text { (menses, OV, L, } \\
\text { next menses) }\end{array}$ & $\begin{array}{l}\text { OV: Reduction in BrainAGE } \\
(-1.27 \text { years) during ovulation }\end{array}$ & $\begin{array}{l}\text { EST (-): } \\
\text { BrainAGE }\end{array}$ \\
\hline $\begin{array}{l}\text { Lisofsky et al. [83], } \\
2015\end{array}$ & $\begin{array}{l}N=21 \mathrm{NC} \\
\text { age range } 22-31 \text { years }\end{array}$ & $\begin{array}{l}\text { Longitudinal } \\
(\mathrm{EF}, \mathrm{LF}, \mathrm{OV}, \mathrm{L})\end{array}$ & $\begin{array}{l}\text { Volume } \\
\text { LF > EF: } \\
\text { R posterior HIPP/thalamus } \\
\text { L posterior HIPP } \\
\text { L thalamus }\end{array}$ & $\begin{array}{l}\text { Volume } \\
\text { EST (+): } \\
\text { L parahippocampal g., } \\
\text { L MFG, } \\
\text { R cerebellum }\end{array}$ \\
\hline $\begin{array}{l}\text { Ossewarde et al. [85], } \\
2013\end{array}$ & $\begin{array}{l}N=28 \mathrm{NC} \\
\text { age range } 18-38 \text { years }\end{array}$ & $\begin{array}{l}\text { Longitudinal } \\
\text { (LF, LL) }\end{array}$ & $\begin{array}{l}\text { Volume } \\
\text { LL > LF: } \\
\text { L dorsolateral amygdala }\end{array}$ & No corr. reported \\
\hline $\begin{array}{l}\text { Protopopescu et al. [84], } \\
2008\end{array}$ & $\begin{array}{l}N=21 \mathrm{NC} \\
\text { age range } 22-35 \text { years }\end{array}$ & $\begin{array}{l}\text { Longitudinal } \\
\text { (LF, LL) }\end{array}$ & $\begin{array}{l}\text { Volume } \\
\text { LF > LL } \\
\text { L occipital (lingual g., } \\
\text { Brodmann area18), } \\
\text { R HIPP/parahippocampus, } \\
\text { L MFG } \\
\text { LL > LF } \\
\text { L superior parietal lobule } \\
\text { (Brodmann area7) } \\
\text { R dorsal basal ganglia } \\
\text { (globus pallidus/putamen) } \\
\text { R MFG/ACC } \\
\text { R thalamus (pulvinar) }\end{array}$ & No corr. reported \\
\hline $\begin{array}{l}\text { De Bondt et al. [90], } \\
2013\end{array}$ & $\begin{array}{l}N=30 \\
n=15 \mathrm{NC} \\
n=15 \mathrm{OC} \\
\text { age range } 18-28 \text { years }\end{array}$ & $\begin{array}{l}\text { Cross-sectional/ } \\
\text { longitudinal } \\
(\mathrm{F}, \mathrm{L})\end{array}$ & $\begin{array}{l}\text { Volume } \\
\text { NC F > L: } \\
\text { R MFG (Brodmann area 6) } \\
\text { L CG R ACC, L MTG } \\
\text { L insula } \\
\text { NC F < L: } \\
\text { R STG } \\
\text { OC inactive > OC active: } \\
\text { L Brodmann area } 6 \\
\text { R PostCG } \\
\text { L caudate (acc) } \\
\text { OC inactive < OC active: } \\
\text { L ACC, L insula } \\
\text { OC active > L NC: } \\
\text { R occipital lobe } \\
\text { R Brodmann area } 6 \\
\text { OC active < L NC: } \\
\text { L FFG }\end{array}$ & $\begin{array}{l}\text { Volume } \\
\text { EST (-): } \\
\text { NC (L): ACC }\end{array}$ \\
\hline
\end{tabular}


Table 2 (continued)

\begin{tabular}{|c|c|c|c|c|}
\hline Study & Groups ( $n$, age) & Design & $\begin{array}{l}\text { Results changes in brain structure } \\
\text { (i.e., volume, brainAGE, ADC, } \\
\text { MD) }\end{array}$ & $\begin{array}{l}\text { Results correlations } \\
\text { hormones }\end{array}$ \\
\hline $\begin{array}{l}\text { Lisofsky et al. [8], } \\
2016\end{array}$ & $\begin{array}{l}N=56(28 \mathrm{NC}, 28 \mathrm{OC}) \\
\text { age range } 16-33 \text { years }\end{array}$ & $\begin{array}{l}\text { Cross-sectional/ } \\
\text { longitudinal } \\
(\mathrm{F}, \mathrm{L})\end{array}$ & $\begin{array}{l}\text { Volume } \\
\text { F > OC: } \\
\text { global GM, } \\
\text { L amygdala, } \\
\text { parahipp complex }\end{array}$ & No corr. reported \\
\hline $\begin{array}{l}\text { Pletzer et al. [87], } \\
2010\end{array}$ & $\begin{array}{l}N=42(n=14 \text { OC } \\
\text { mean age: } 25.9 \text { years, } \\
n=14 \text { NC, } \\
\text { mean age: } 23.2 \text { years, } \\
n=14 \text { men } \\
\text { mean age: } 25.3 \text { years }\end{array}$ & $\begin{array}{l}\text { Cross-sectional/ } \\
\text { longitudinal } \\
(\mathrm{EF}, \mathrm{ML})\end{array}$ & $\begin{array}{l}\text { Volume } \\
\text { EF > ML } \\
\text { R FFG, parahipp. g. } \\
\text { OC > NC (EF phase) } \\
\text { L/R PFC, L/R PreCG L/R PostCG, } \\
\text { L/R ACC, L/R SMA, L insula, L } \\
\text { rolandic operculum, L IFG, } \\
\text { L SMG, L IPG, L/R MTG, } \\
\text { L/R MSTG, R ICG, R FFG, } \\
\text { R temporal pole, R lingual g., R } \\
\text { parahipp. g. } \\
\text { OC > NC (ML phase) } \\
\text { L/R PFC, L/R PreCG, L/R ACC, } \\
\text { L rolandic operculum } \\
\text { L PostCG, L SMG } \\
\text { L/R ITG, L/R Inferior OCG } \\
\text { L/R MTG, R Temporal pole, } \\
\text { R lingual g., R parahipp g., } \\
\text { R FFG, R HIPP, L/R cerebellum }\end{array}$ & No corr. reported \\
\hline $\begin{array}{l}\text { Petersen et al. [88], } \\
2015\end{array}$ & $\begin{array}{l}N=90 \\
n=44 \text { OC } \\
\text { [22 inactive pill week, } \\
22 \text { active pill week], } \\
n=46 \mathrm{NC} \\
[21 \mathrm{~F}, 25 \mathrm{~L}])\end{array}$ & Cross-sectional & $\begin{array}{l}\text { Cortical thickness } \\
\text { NC (F and L phase })>\text { OC } \\
\text { L PCC } \\
\text { L caudal ACC } \\
\text { L insula } \\
\text { L/R lateral OFC } \\
\text { L/R medial OFC }\end{array}$ & $\begin{array}{l}\text { Cortical thickness } \\
\text { PROG (+): L insula } \\
\text { EST(+): L lateral OFC }\end{array}$ \\
\hline
\end{tabular}


Table 2 (continued)

\begin{tabular}{|c|c|c|c|c|}
\hline Study & Groups ( $n$, age) & Design & $\begin{array}{l}\text { Results changes in brain structure } \\
\text { (i.e., volume, brain AGE, ADC, } \\
\text { MD) }\end{array}$ & $\begin{array}{l}\text { Results correlations } \\
\text { hormones }\end{array}$ \\
\hline $\begin{array}{l}\text { Witte et al. [94], } \\
2010\end{array}$ & $\begin{array}{l}N=34 \\
n=17 \mathrm{NC}[\mathrm{MF}] \\
n=17 \text { men, } \\
\text { mean age: } 26.6 \text { years }\end{array}$ & Cross-sectional & & $\begin{array}{l}\text { EST (+): } \\
\text { L IFG volume } \\
\text { TEST (-): } \\
\text { L IFG volume } \\
\text { PROG (-): } \\
\text { R temporal pole vol- } \\
\text { ume }\end{array}$ \\
\hline $\begin{array}{l}\text { White matter } \\
\text { Baroncini et al. [93], } \\
2009\end{array}$ & $\begin{array}{l}N=20 \\
n=10 \text { OC } \\
n=10 \text { men } \\
\text { age range } 19-25 \text { years }\end{array}$ & $\begin{array}{l}\text { Cross-sectional/ } \\
\text { Longitudinal } \\
\text { (active vs inactive) }\end{array}$ & $\begin{array}{l}\text { OC inactive }>\text { OC active } \\
\text { ADC hypothalamus }\end{array}$ & \\
\hline $\begin{array}{l}\text { Barth et al. [91], } \\
2016\end{array}$ & $\begin{array}{l}n=1 \mathrm{NC} \\
\text { age: } 32 \text { years }\end{array}$ & $\begin{array}{l}\text { Single subject case } \\
\text { study/ } \\
\text { longitudinal ( } 30 \\
\text { measurements) }\end{array}$ & $\begin{array}{l}\text { OV: } \\
\text { Peak FA }\end{array}$ & $\begin{array}{l}\text { EST }(+) \\
\text { FA R/L HIPP } \\
\text { GM R HIPP }\end{array}$ \\
\hline
\end{tabular}

* Same study population. Studies investigating the influence of sex hormones on changes in brain structure across the menstrual cycle or oral contraceptive intake. Only significant results are listed. MNI coordinates can be found in online supplementary 1. L, left; $\mathrm{R}$, right; ADC, apparent diffusion coefficient; NC, naturally cycling; OV, ovulation; L, luteal; EST, estradiol; (-), negative correlation; EF, early follicular; LF, late follicular; HIPP, hippocampus; (+), positive correlation; MFG, middle frontal gyrus; LL, late luteal; OC, oral contraceptive intake; F, follicular; ACC, anterior cingulate; MTG, middle temporal gyrus; MD, mean diffusivity; LH, luteinizing hormone; PROG, progesterone; ML, midluteal; PreCG, precentral gyrus; PostCG, postcentral gyrus; OCG, occipital gyrus; parahipp g, parahippocampal gyrus; FFG, fusiform gyrus; PCC, posterior cingulate cortex; FFA, fusiform face area; PPA, parahippocampal gyrus; GM, gray matter; PROG, progesterone; SMG, supramarginal gyrus; IFG, inferior frontal gyrus; STG, superior temporal gyrus; PFC, prefrontal cortex; ITG, inferior temporal gyrus; TEST, testosterone; MD, mean diffusivity; FA, fractional anisotropy; OFC, orbitofrontal cortex.

found between testosterone and volume of the right diencephalic structures including hypothalamus, mammilary bodies and ventral thalamus [77].

\section{White Matter}

Recent studies indicate that development of white matter during puberty may be partially affected by levels of sex hormones [73, 78, 79]. Perrin et al. [78] showed an overall increase in white matter volume in a cross-sectional study with an age range of $10-18$ years. Peper et al. [73] reported a positive association between LH and increased overall white matter volume as well as density in specific regions, including the left cingulum, splenium of the corpus callosum, MTG and right superior frontal gyrus, Paus et al. [79] found a positive association with testosterone and the relative/absolute white matter to gray 
matter ratio. Herting et al. [81] reported a positive correlation of testosterone with the precentral gyrus white matter. For estradiol a negative association with the fractional anisotropy of the right angular gyrus white matter and the superior longitudinal fasciculus was found [81] Table 1.

\section{Menstrual Cycle/Oral Contraception}

We identified a total number of thirteen studies that measured NC women or women taking OCs with respect to global or regional GMV (for details, please see Table 2).

\section{Grey Matter Volume}

Hagemann et al. [82] reported increased global GMV during OV compared to EF phase (EF; OV > EF) in a longitudinal study including $7 \mathrm{NC}$ women.

Most prominent changes in brain volume have been reported for hippocampal volume across the menstrual cycle. Lisofsky et al. [83] measured NC women at 4 times during EF, LF, OV and luteal phase (L) and observed an increase in bilateral hippocampal volume in the late compared to the EF phase (EF > L). Similarly, Protopopescu et al. [84] reported a regional right anterior hippocampal volume increase in the LF compared to the LL phase (LF $>$ LL) in women measured twice during their menstrual cycle.

Regarding amygdala volume, Ossewaarde et al. [85] measured NC women twice during the LF and LL phase and reported a significantly larger amygdala volume in the LL compared to the LF phase (LL > LF). De Bondt et al. [86] reported higher insula volume during the ovulatory phase compared to the luteal phase $(\mathrm{OV}>\mathrm{L})$. Notably, Pletzer et al. [87] gave first evidence for a GMV increase in a cluster including fusiform gyrus/parahippocampal gyrus during the EF compared to the ML phase (EF > ML).

Investigating the effect of OC intake on brain morphology, Lisofsky et al. [8] compared NC women to women before and 3 months after starting OC intake and reported reduced GMV of the amygdala/parahippocampal complex as a whole brain group $\times$ time interaction effect in OC-users compared to $\mathrm{NC}$ women measured during the EF phase (L > OC). Also, Petersen et al. [88] showed reduced GMV in OC-users compared to $\mathrm{NC}$ women measured during the follicular and luteal phase $(\mathrm{NC}>$ $\mathrm{OC})$, particularly including volume changes in the left insula, caudal anterior cingulate, and bilateral orbitofrontal cortex. Increased GMV has also been reported in users of anti-androgenic OCs compared to EF women in bilateral fusiform gyri, fusiform face area, para-hippocampal place area (PPA) and cerebellum (OC[lowT] >EF; [89]). De Bondt et al. [90] conducted one of the most comprehensive studies of OC use so far, also considering possible differences between the active intake weeks, during which endogenous hormones are suppressed, and the pill-free week. Increases in grey matter during the active compared to the pill-free week were found in the mid-frontal gyrus (Brodmann area 6), posterior central gyrus, caudate nucleus, insula and ACC [90].

\section{White Matter}

So far, only a few studies have investigated white matter changes across the menstrual cycle or OC use. Barth et al. [91] provided an interesting single subject case study, measuring one woman repeatedly across 2 menstrual cycles and reported a peak in fractional anisotropy at OV. Moreover, in the fornix higher mean diffusivity (MD) values were reported for women using OCs in comparison to NC women during the follicular phase [92]. When comparing women in the active OC phase to the pill-free week, higher apparent diffusion coefficient values were found in the hypothalamus during the active OC phase [93].

Direct correlation analyses of hormonal levels were reported in 6 studies. Estradiol showed a significant negative correlation with ACC volumes in luteal women [90] and $\mathrm{MD}$ of the fornix in $\mathrm{NC}$ women and women taking OCs [92]. In a single case study, a positive correlation of estradiol with hippocampal grey matter and FA was shown across the menstrual cycle [91]. For progesterone, Petersen et al. [88] found a positive relationship with thickness of the left insula, Witte et al. [94] a negative relationship with the volume of the right temporal pole, and Hagemann et al. [82] a significant negative association with overall change in grey matter volume. Only one study reported a significant association of testosterone levels with the left inferior frontal gyrus (IFG) in NC women [94]. A negative correlation between levels of LH and MD of the fornix was observed [92].

Using the BrainAGE-approach, Franke et al. [95] analysed and aggregated structural patterns of aging based on MRI data and compared the given age value to the subject's actual age [95-97]. When measuring a sample of $\mathrm{NC}$ women during their menses, OV, luteal phase and next menses, women during OV showed a significant reduction in BrainAGE of around -1.27 years between menses and OV [95] Table 2. 
Table 3. Pregnancy and postpartum period

\begin{tabular}{|c|c|c|c|}
\hline $\begin{array}{l}\text { Hoekzema et al. [101], } \\
2017^{*}\end{array}$ & $\begin{array}{l}N=81 \\
n=25 \text { primiparous women } \\
\text { mean age: } 33.36 \\
n=20 \text { nulliparous women } \\
\text { mean age: } 31.10, \\
n=19 \text { first time fathers, } \\
\text { mean age: } 35.21, \\
n=17 \text { men without children } \\
\text { mean age: } 31.64\end{array}$ & $\begin{array}{l}\text { Prospective longitudinal } \\
\text { (pre vs. post-pregnancy)/ } \\
\text { cross-sectional }\end{array}$ & $\begin{array}{l}\text { Control > post-pregnancy } \\
\text { L/R STS, L/R MTG, L/R STG, L/R Precuneus, } \\
\text { L/R ACC, L/R PCC, } \\
\text { L inferior and medial OFG, } \\
\text { L/R IFG, L Insula, L MFG, L SFG, } \\
\text { L/R FFG, L/R ITG, L HIPP, } \\
\text { parahippocampal g. } \\
\text { PP < Pre-pregnancy } \\
\text { L/R STS, L/R ITG, L/R MTG, L/R STG, L/R } \\
\text { AG, L/R Parahippocampal g., } \\
\text { L/R Hippocampus, L/R PCG, } \\
\text { L/R Superior MFC, L/R ACC, } \\
\text { L/R Medial OFC, L/R IFC, L/R MFC, L/R } \\
\text { SFC, L/R Precuneus, L Inferior OFC, L } \\
\text { Insula, L/R inferior OFC, R temporal pole, } \\
\text { L/R Posterior cerebellum }\end{array}$ \\
\hline $\begin{array}{l}\text { Hoekzema et al. [103], } \\
2020^{*}\end{array}$ & $\begin{array}{l}N=45 \\
n=25 \text { primiparous } \\
n=20 \text { nulliparous } \\
\text { mean age: } 32.85 \text { years }\end{array}$ & $\begin{array}{l}\text { Cross-sectional/ } \\
\text { Prospective longitudinal } \\
\text { (pre vs. post-pregnancy) }\end{array}$ & $\begin{array}{l}\text { Control > pregnant } \\
\text { R ventral striatum }\end{array}$ \\
\hline $\begin{array}{l}\text { Kim et al. [106], } \\
2010\end{array}$ & $\begin{array}{l}N=19 \text { mothers } \\
n=8 \text { multiparous } \\
n=11 \text { primiparous } \\
\text { mean age }=33.27\end{array}$ & $\begin{array}{l}\text { Longitudinal } \\
\text { T1: Postpartum } \\
\text { (2-4 weeks) } \\
\text { T2: Postpartum } \\
\text { (3-4 months) }\end{array}$ & $\begin{array}{l}\text { PP T2 > T1 } \\
\text { L/R IPL, L/R SPL, L/R precuneus } \\
\text { MFG, PreCG, CG, L IFG, R thalamus, } \\
\text { L/R hypothalamus, L/R substantia } \\
\text { nigra, L/R caudate body, L/R caudate } \\
\text { head, L/R mammillary body, R } \\
\text { amygdala, R putamen, R medial } \\
\text { globus pallidus, R ACG, } \\
\text { R parahippocampal g., R Insula, L/R } \\
\text { cerebellum }\end{array}$ \\
\hline $\begin{array}{l}\text { Oatridge et al. [104], } \\
2002\end{array}$ & $\begin{array}{l}N=14 \\
n=9 \text { healthy } \\
\text { pregnant/postpartum, } \\
n=5 \text { pregnant with } \\
\text { preeclampsia } \\
\text { mean age: } 31 \text { years }\end{array}$ & $\begin{array}{l}\text { Cross-sectional/ } \\
\text { longitudinal/ } \\
\text { (pregnancy 2nd trimester/ } \\
6 \text { weeks PP (preeclampsia/6 } \\
\text { months postpartum) }\end{array}$ & $\begin{array}{l}\text { Pregnancy }<6 \text { months PP } \\
\text { Brain volume } \\
\text { Pregnancy }>6 \text { months PP } \\
\text { Ventrical size }\end{array}$ \\
\hline
\end{tabular}


Table 3 (continued)

\begin{tabular}{|c|c|c|c|}
\hline Study & Groups & Design & Results \\
\hline Dinc et al. [100], 1998 & $\begin{array}{l}N=96 \\
\text { Group 1: } n=10 \\
\text { pregnant } 2 \text { nd } \\
\text { Trimester } \\
\text { Group 2: } n=14 \\
\text { 3rd trimester } \\
\text { Group 3: } n=32 \\
\text { 0-3 days PP } \\
\text { Group 4: } n=10 \\
\text { 4 days }-2 \text { months PP } \\
\text { Group 5: } n=12 \\
\text { 2-6 months PP } \\
\text { Group 6: } n=18 \text { controls } \\
\text { age range } 20-38 \text { years }\end{array}$ & $\begin{array}{l}\text { Cross-sectional/ } \\
\text { longitudinal }\end{array}$ & $\begin{array}{l}\text { ROI pituitary gland } \\
\text { Volume: } \\
\text { Group } 2 / 3>\text { Group } 1 / 4 / 5 / 6 \\
\text { Group } 3>\text { Group 2; } \\
\text { Group } 1>\text { Group } 4 / 5 \text {, } \\
\text { Group } 4>\text { Group } 5 / 6 \\
\text { No significant differences between groups } 5 \\
\text { and } 6\end{array}$ \\
\hline Gonzalez et al. [98], 1988 & $\begin{array}{l}N=55 \\
\text { Group 1: } n=10,<12 \mathrm{GW}, \\
\text { Group 2: } n=11,13-26 \mathrm{GW}, \\
\text { Group 3: } n=11, \geq 27 \mathrm{GW} \\
\text { Group 4: } n=20 \text { nulliparous } \\
\text { age range } 16-30 \text { years }\end{array}$ & Cross-sectional & $\begin{array}{l}\text { ROI pituitary gland } \\
\text { Volume: } \\
\text { Group } 1 / 2 / 3>\text { Group } 4 \\
\text { Group } 3>\text { Group } 1 / 2 \\
\text { Groups } 1 \text { and } 2 \text { did not differ } \\
\text { significantly }\end{array}$ \\
\hline
\end{tabular}

* Same study population. Studies investigating the influence of sex hormones on changes in brain structure. Only significant findings are listed. MNI coordinates can be found in online supplementary 1. L, left; R, right; PFC, prefrontal cortex; STS, superior temporal sulcus; MTG, middle temporal gyrus; STG, superior temporal gyrus; ACC, anterior cingulate cortex; PCC, posterior cingulate cortex; OFG, orbitofrontal gyrus; PP, postpartum; IFG, inferior frontal gyrus; MFG, middle frontal gyrus; SFG, superior frontal gyrus; FFG, fusiform gyrus; ITG, inferior temporal gyrus; HIPP, hippocampus; g., gyrus; AG, angular gyrus; PP, postpartum; IPL, inferior parietal lobe; SPL, superior parietal lobe; PreCG, precentral gyrus; CG, cingulate gyrus; ACG, anterior cingulate gyrus; PCG, posterior cingulate gyrus; MFC, medial frontal cortex; OFC, orbitofrontal cortex; IFC, inferior frontal cortex; SFC, superior frontal cortex; GW, gestational week.

\section{Pregnancy and Postpartum Period}

In total 10 studies were found including effects of pregnancy and postpartum on brain volume (for details, please see Table 3).

Even though pregnancy is an important hormonal transition period for women, only a few studies have investigated the influence of pregnancy on the brain. Several earlier studies showed increases up to $136 \%$ in the pituitary gland at the end of pregnancy compared to nulliparous women [98-100] followed by return to original size by 2 weeks to 6 months postpartum $[98,99]$. Over the course of pregnancy, a continuous progression of size of the pituitary gland corresponding with gestational week that did not exceed $10 \mathrm{~mm}$ could be observed [98]. The highest increase in size was reported at 3 days postpartum [100].

A recent study of Hoekzema et al. [101] reported longer lasting reductions in GMV comparing pre-pregnancy to shortly after delivery and up to 2 years postpartum in first-time pregnant women. Authors reported long lasting reduction in GMV, which was most pronounced for areas important for social cognition, that is, the anterior and posterior midline structures, bilateral prefrontal cortex and bilateral temporal cortex. Furthermore, wom- 
Table 4. Menopause

\begin{tabular}{|c|c|c|c|c|}
\hline Study & Groups & Medication/dose & Design & Results \\
\hline \multicolumn{5}{|c|}{ Randomized, placebo-controlled trials } \\
\hline $\begin{array}{l}\text { Albert et al. } \\
\text { [107], } 2017\end{array}$ & $\begin{array}{l}N=78 \\
n=33,2 \text { mg E2 } \\
\text { mean age }=61.9 \text { years } \\
n=21,1 \text { mg E2 } \\
\text { mean age }=55.5 \text { years, } \\
n=21, \text { Placebo } \\
\text { mean age }=56.8 \text { years }\end{array}$ & $\begin{array}{l}2 \mathrm{mg} \mathrm{E} 2 \\
1 \mathrm{mg} \mathrm{E} 2 \\
\text { Placebo }\end{array}$ & $\begin{array}{l}\text { Prospective (pre vs. post)/ } \\
\text { randomized, placebo controlled } \\
\text { trial (blinded/unblinded) }\end{array}$ & $\begin{array}{l}2 \mathrm{mg}>1 \mathrm{mg} \text {, placebo: } \mathrm{L} / \mathrm{R} \\
\text { HIPP volume }\end{array}$ \\
\hline $\begin{array}{l}\text { Kantarci et al. } \\
{[116], 2016}\end{array}$ & $\begin{array}{l}N=95 \\
n=29, \text { CEE } \\
\text { mean age }=53 \text { years, } \\
n=30, \text { E } 2 \\
\text { mean age }=53 \text { years, } \\
n=36, \text { Placebo } \\
\text { mean age }=53 \text { years }\end{array}$ & $\begin{array}{l}\text { oral CEE ( } 0.45 \mathrm{mg} \text { /day; premarin) } \\
\text { transdermal E2 (skin patch, } 50 \mathrm{mg} / \text { day) }\end{array}$ & $\begin{array}{l}\text { Prospective (pre vs. post)/ } \\
\text { randomized, double-blind, } \\
\text { placebo-controlled trial }\end{array}$ & $\begin{array}{l}\mathrm{CEE}>\text { placebo: ventricular } \\
\text { volume }\end{array}$ \\
\hline $\begin{array}{l}\text { Zhang et al. } \\
\text { [115], 2016* }\end{array}$ & $\begin{array}{l}N=1,356 \\
n=254, \text { CEE-alone group } \\
n=420, \text { CEE + MPA group } \\
n=691, \text { placebo group } \\
\text { age: } 65 \text { and older }\end{array}$ & $\begin{array}{l}0.625 \mathrm{mg} / \text { day of CEE-alone (after } \\
\text { hysterectomy) or CEE in combination } \\
\text { with } 2.5 \mathrm{mg} \text { MPA per day (with intact } \\
\text { uterus) }\end{array}$ & $\begin{array}{l}\text { Retrospective/randomized and } \\
\text { placebo } \\
\text { controlled trial }\end{array}$ & $\begin{array}{l}\text { Users < placebo: L/R ACC, L } \\
\text { MSFG, L SFG L/R MCC, L } \\
\text { OFC, L/R g. rectus CEE- } \\
\text { alone < placebo L/R ACC, } \\
\text { L/R MSFG, R MCC }\end{array}$ \\
\hline \multicolumn{5}{|c|}{ Cross-sectional design } \\
\hline $\begin{array}{l}\text { Boccardi et al. } \\
{[110], 2006}\end{array}$ & $\begin{array}{l}N=40 \\
n=16, \text { current } \mathrm{ET} \\
\text { mean age }=57.4 \text { years } \\
n=7, \text { past ET } \\
\text { mean age }=63.4 \text { years } \\
n=17 \text {, never-users } \\
\text { mean age }=60.8 \text { years }\end{array}$ & $\begin{array}{l}\text { Estraderm, } 0.05 / 0.1 \mathrm{mg} \text { of E2 per day, } \\
\text { transdermal) }\end{array}$ & Retrospective/cross-sectional & $\begin{array}{l}\text { ET+ > ET-: L/R cerebellum, } \\
\text { R MTG, L IFG } \\
\text { Current > past: L/R } \\
\text { cerebellum, R MTG, L IFG } \\
\text { (whole brain) }\end{array}$ \\
\hline $\begin{array}{l}\text { Eberling et al. } \\
\text { [112], 2004 }\end{array}$ & $\begin{array}{l}N=30 \\
n=15, \mathrm{ET}+ \\
\text { mean age }=67.3 \text { years } \\
n=15, \mathrm{ET}- \\
\text { mean age }=66.5 \text { years } \\
n=10, \mathrm{TAM}+ \\
\text { mean age }=64.7 \text { years }\end{array}$ & $\begin{array}{l}\text { Unopposed estrogen (mainly Premarin). } \\
\text { No dose indicated } \\
\text { Tamoxifen (treatment for breast cancer) }\end{array}$ & Retrospective/cross-sectional & $\begin{array}{l}\text { ET+ > ET-: L IFC and } \\
\text { temporal c. and R STG } \\
\text { (whole brain); no HIPP } \\
\text { differences (ROI) }\end{array}$ \\
\hline $\begin{array}{l}\text { Erickson et al. } \\
\text { [109], } 2005\end{array}$ & $\begin{array}{l}N=43 \\
n=16, \text { current } \mathrm{MHT} \\
\text { age }=68.9 \text { years } \\
n=14, \text { past } \mathrm{MHT} \\
\text { age }=66.2 \text { years } \\
n=13, \text { never users } \\
\text { age }=68.4 \text { years }\end{array}$ & $\begin{array}{l}\text { Conjugated equine estrogens (Premarin) } \\
\text { (current MHT: 13; past MHT: 10) }\end{array}$ & Retrospective/cross-sectional & $\begin{array}{l}\text { MHT+ > MHT-: frontal, } \\
\text { prefrontal, and temporal } \\
\text { regions (whole brain) }\end{array}$ \\
\hline $\begin{array}{l}\text { Erickson et al. } \\
\text { [117], } 2010\end{array}$ & $\begin{array}{l}N=102 \\
n=65, \mathrm{MHT}+ \\
\text { age }=65.4 \text { years } \\
n=37, \text { MHT }- \\
\text { age }=69.5 \text { years }\end{array}$ & $\begin{array}{l}63 \% \text { of MHT users received unopposed } \\
\text { CEEs, } 29 \% \text { received CEE in combination } \\
\text { with MPA and the remainder received PEs }\end{array}$ & Retrospective/cross-sectional & $\begin{array}{l}\text { Longer time between onset } \\
\text { of menopause and treatment } \\
\text { initiation was negatively } \\
\text { associated with R/L HIPP } \\
\text { volume (regression analysis) }\end{array}$ \\
\hline
\end{tabular}




\begin{tabular}{|c|c|c|c|c|}
\hline Study & Groups & Medication/dose & Design & Results \\
\hline $\begin{array}{l}\text { Greenberg et al. } \\
{[113], 2006}\end{array}$ & $\begin{array}{l}N=92 \\
n=41, \text { MHT }+ \\
\text { age }=70 \text { years } \\
n=51, \text { MHT }- \\
\text { age }=71 \text { years }\end{array}$ & $\begin{array}{l}\text { Diverse medication and doses (estradiol } \\
\text { only, estradiol + progestin) }\end{array}$ & Retrospective/cross-sectional & $\begin{array}{l}\text { MHT+< MHT-: L putamen } \\
\text { MHT+ > MHT-: R putamen } \\
\text { (ROI) }\end{array}$ \\
\hline $\begin{array}{l}\text { Kim et al. } \\
{[118], 2018}\end{array}$ & $\begin{array}{l}N=40 \text { women } \\
n=20 \text {, pre-menopausal } \\
\text { mean age: } 39.9 \text { years } \\
n=20 \text {, post-menopausal } \\
\text { mean age: } 55.7 \text { years }\end{array}$ & $\begin{array}{l}\text { Post-menopausal: no hormone intake } 1 \\
\text { month before study }\end{array}$ & Cross-sectional & $\begin{array}{l}\text { Pre-menopause > post- } \\
\text { menopause (adjusted for } \\
\text { age): L SMA, L IFG, R } \\
\text { olfactory c., and R STG } \\
\text { EST (+): L SMA, L IFG and } \\
\text { R STG } \\
\text { FSH (-): L SMA, L IFG; R } \\
\text { STG }\end{array}$ \\
\hline $\begin{array}{l}\text { Lord et al. } \\
{[111], 2008}\end{array}$ & $\begin{array}{l}N=41 \\
n=16, \text { CEE } \\
\text { age }=59 \text { years } \\
n=10, \text { past users } \\
\text { age }=66 \text { years } \\
n=15, \text { never-users } \\
\text { age }=60 \text { years }\end{array}$ & $\begin{array}{l}\text { MHT-users were using either CEE or E2 } \\
\text { therapy (oral or transdermal) }\end{array}$ & Retrospective/cross-sectional & $\begin{array}{l}\text { Current MHT > past MHT } \\
\text { and never-users: } \\
\text { R HIPP volume (ROI) } \\
\text { Longer use of HRT } \\
\text { negatively linked to total } \\
\text { HIPP volume }\end{array}$ \\
\hline
\end{tabular}

* Data come from the same study. Studies investigating the impact of menopausal hormone therapy (MHT) on brain structure. Only significant findings are listed. MNI coordinates can be found in online supplementary 1. E2, estradiol; L, left; R, right; HIPP, hippocampus; CEE, conjugated equine estrogen; MPA, medroxyprogesterone acetate; ACC, anterior cingulate cortex; MSFG, medial superior frontal gyrus; SFG, superior frontal gyrus; MCC, medial cingulate cortex; OFC, orbitofrontal gyrus; g., gyrus; IFC, inferior frontal cortex; ET, estrogen treatment; MTG, middle temporal gyrus; IFG, inferior frontal gyrus; c., cortex; MHT, menopause hormone treatment; SMA, supplementary motor area; STG, superior temporal gyrus; (+), positive correlation; (-), negative correlation.

en having undergone pregnancy could be classified correctly by $95.6 \%$ using measures of average grey matter change. The greatest predictors for a past pregnancy were reductions in the right MTG, IFG and posterior cingulate cortex. Interestingly, an overlap between areas with reductions and the Theory-of-Mind network could be observed (for review please see [102]). In a follow-up study of the same study population a significant reduction in the right ventral striatum was found. No pregnancy-related changes occurred for the dorsal striatum [103]. When women are followed from pregnancy into the postpartum period the picture is seemingly different. An earlier study supports the notion of increase in grey matter and reduction in ventricular size from delivery to 6 months postpartum [104]. In line with previously reported transient changes, Luders et al. [105] conducted anatomical brain scans of women in the early postpartum phase 1-2 days after delivery and again during the late postpartum at 4-6 weeks after delivery. Again using the BrainAGE algorithm [95] a rejuvenating effect was noted, with a reduction of 5 years of age observed from early to late postpartum. In a longitudinal study Kim et al. [106] assessed changes in the postpartum period 2-3 weeks and again 3-4 months after delivery. Authors reported increases in grey matter of the prefrontal cortex, parietal lobes, and midbrain areas including the right amygdala, hippocampus, substantia nigra at the second time point Table 3.

\section{Menopause}

In total, 12 studies investigating the influence of sex hormones on changes during and after menopause as well as hormone replacement therapy were included in this review (for details, please see Table 4).

One of the most comprehensive studies, investigating changes in brain volume before and after hormone intake, was conducted by Albert et al. [107]. Two doses of oestrogen ( 2 vs. $1 \mathrm{mg}$ daily) or a placebo were administered to healthy post-menopausal women for 3 months in a longitudinal design. The authors reported a dosedependent increase of bilateral hippocampal volume following 3 months of treatment. Eberling et al. [108] conducted a retrospective cross-sectional study comparing the hippocampal volumes of postmenopausal women receiving ET to postmenopausal women not receiving treatment. Here the authors reported increased 


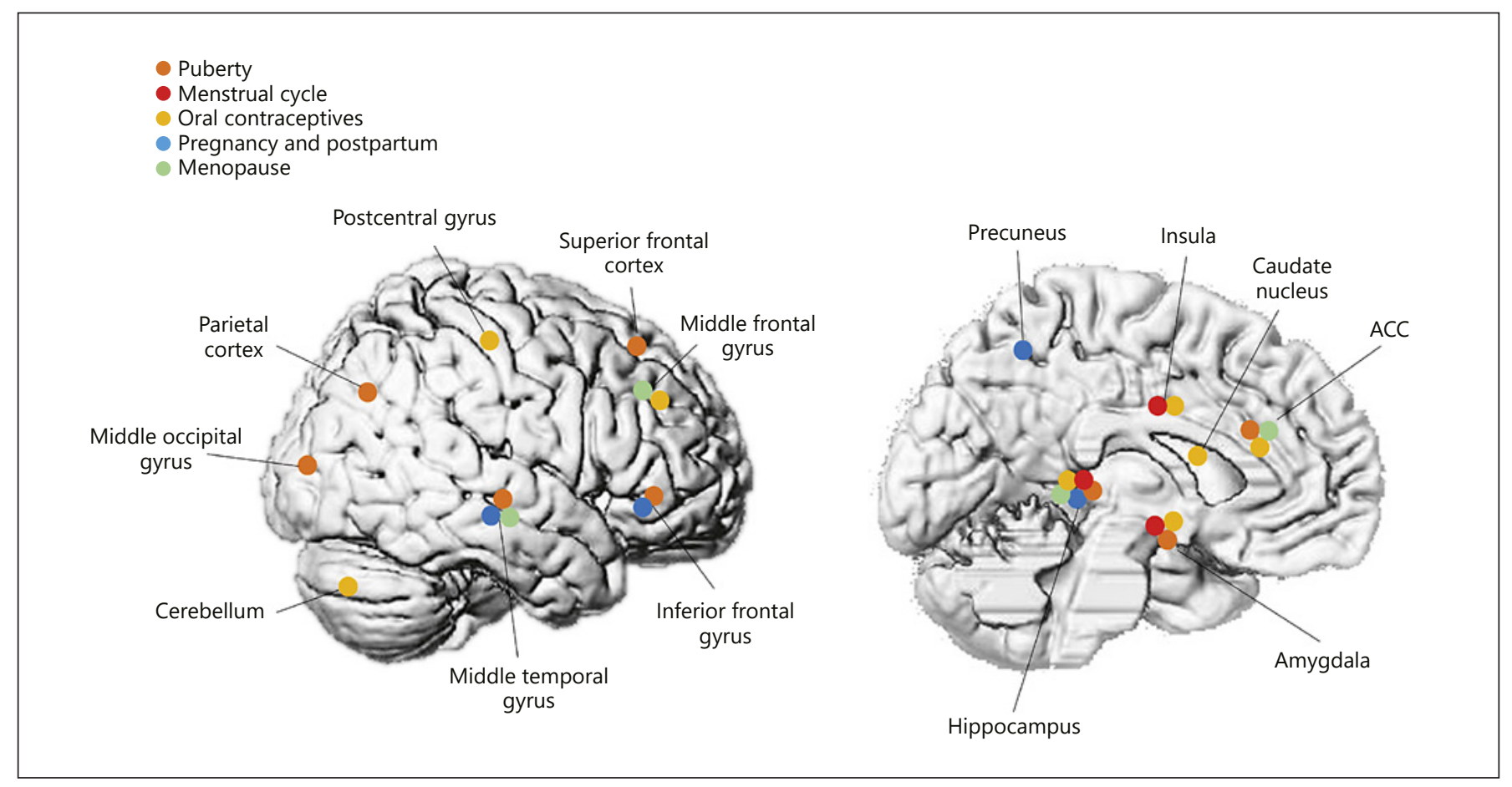

Fig. 1. Volumetric grey matter changes during hormonal transition periods. ACC, anterior cingulate.

right anterior hippocampal volumes in users of ET. To evaluate whether past use of MHT may also have beneficial effects on brain volume, Erickson et al. [109] incorporated both current users of MHT, past users of MHT and women who have never used MHT from the same age group. While current and past users of MHT did not differ regarding brain volumes, both of these groups had significantly larger volumes of anterior hippocampus, prefrontal and temporal areas. Similarly, increased volumes of cerebellum and the amygdaloidhippocampal complex in current and past users of MHT to never users [110] and larger right hippocampal volumes [111] were found. Interestingly, past users of MHT had even larger volumes than current users of MHT [110]. All of these studies indicate a significant effect of MHT in the direction of volume increases in several areas, with the hippocampus being most consistent. However, there are also studies reporting decreases of brain volume following MHT: while Eberling et al. [112] found no difference in hippocampal volume between healthy users of MHT and non-users, Greenberg et al. [113] showed reduced total GMV and increased cerebrospinal fluid in users of MHT in a retrospective cross-sectional study. In this case, however, the hippocampus was not affected differently between groups.
Another study by Resnick et al. [114] observed a reduction of frontal lobe and hippocampal volume in MHT users compared to the placebo group in a cross-sectional study. Furthermore, Zhang et al. [115] reported reduced volume of the anterior cingulate cortex and the orbitofrontal cortex in MHT users compared to a placebo group, while Kantarci et al. [116] showed expansion of the ventricular volume in MHT-users compared to a placebo group, indicating reduced brain volume. A negative association between time since menopause onset and hippocampal volume was reported [117]. Kim et al. [118] found higher volume of the supplementary motor area (SMA), IFG, olfactory cortex, and superior temporal gyrus (STG) in premenopausal women compared to postmenopausal women that did not undergo hormone replacement therapy for at least 1 month. Moreover, a positive association of estradiol with grey matter volume of the SMA, IFG, and STG and a negative correlation of FSH with grey matter volume of the SMA, IFG, and STG [118] (Table 4 for a summary of studies investigating the impact of postmenopausal MHT). Taken together, results derived from the abovementioned studies are quite diverse, that is, show volume increases, decreases beneficial, detrimental or null-effects of MHT on grey matter Figure 1. 


\section{Discussion}

The aim of this review was to investigate the impact of sex hormones (i.e., estradiol, progesterone, testosterone and $\mathrm{LH}$ ) on brain structure during hormonal transition periods across a women's life span including puberty, the reproductive period (menstrual cycle, hormonal contraceptive, pregnancy, postpartum period) and menopausal transition.

\section{Puberty}

Studies assessing puberty found an overall reduction in grey matter and region-specific reductions in prefrontal, parietal and middle temporal areas [74-76]. Even though the exact mechanisms are yet to be clarified, a decrease in grey matter is mainly associated with the process of synaptic pruning [119, for review please see 120]. After an overproduction of neurons postnatally, a decrease during puberty occurs until adult levels are reached. These reductions seem to be correlated with changes in hormonal levels, with estradiol showing greater influence in girls [78]. At the same time, an increase in white matter through myelination can be observed [121]. When comparing studies, results are inconclusive regarding the influence of sex hormone levels on specific brain areas. Here, several studies report a negative association of global or specific grey matter with estradiol $[75,76,78]$ and testosterone $[73,76]$, some observed positive associations of hormonal levels and total brain volume [77-79], while again others found no correlation [72, 75, 79]. With regard to whole brain white matter, positive correlations with $\mathrm{LH}$ and testosterone levels have been observed $[73,78]$. One reason for these contradicting results may be age and pubertal stage differences between studies; making the comparison of study results difficult and promoting rather speculative assumptions of hormonal influences on brain structure and morphology.

On a behavioural level, the rise in sex hormones is associated with changes in social and motivational tendencies [122]. Adolescents start to invest more time in their peer bonding and in establishing relationships outside of the family context. Studies showed an increased tendency of sensation seeking and involvement in high-risk behaviour [123-125]. As a consequence, problems of controlling emotions or delaying short-term gratifications in order to pursue long-term goals appear. One explanation for this change in behaviour can be linked to brain development, as areas found in the results are relevant for cognitive control, that is, the prefrontal cortex and parietal area, are restructured and myelinated during puberty [ 74 , $75,77,124]$.

Shaping of the Female Brain by Sex Hormones

\section{Menstrual Cycle and OC Use}

Currently only few studies are available that specifically investigated the influence of sex hormones on brain structure during the menstrual cycle or women taking OCs. Across the menstrual cycle, grey matter volumes of the hippocampus, the amygdala as well as temporal- and parietal lobes seem to be most consistently affected by fluctuating hormones. Other structures, for example, the orbitofrontal gyrus, insula, cerebellum, mid-frontal gyrus (Brodmann area 6), ACC were mentioned repeatedly, albeit with inconsistent patterns. Some studies including women using oral contraception reported localized decreases in cortical thickness, for example, orbitofrontal cortex, insula, caudal anterior cingulate cortex and orbitofrontal cortex and decrease of grey matter in specific brain regions, that is, left amygdala and anterior parahippocampal gyrus. Contrarily, other studies reported increases in volume of the fusiform gyrus, PPA, cerebellum, temporal and parietal gyrus when comparing OC to NC women during the follicular phase and luteal phase. Across different phases of the menstrual cycle, studies report differences in emotional processing and emotion recognition [126-128]. Thereby, women in the EF phase showed the highest accuracy in detecting angry as well as sad emotional expressions when compared to women in the luteal phase [126]. A negative correlation between progesterone and recognition accuracy was observed [127]. With regard to affect, women often report better mood ratings during the follicular phase than the luteal phase [129]. Women suffering from premenstrual dysphoric disorder, experience increases in anxiety and depressive symptoms during the LL phase and deficits in emotion recognition as well as a tendency to misjudge neutral faces as negative [130], (for review please see [34]). Whether the structural changes reported in the studies contribute to these cycle-dependent behavioural differences needs to be further investigated.

For OC use localized decreases in cortical thickness, for example, of the orbitofrontal cortex, insula, caudal anterior cingulate cortex and orbitofrontal cortex as well as grey matter volume reduction in specific brain regions, (for review please see [131]). Increases in the fusiform gyrus, PPA, cerebellum, temporal and parietal gyrus when comparing $\mathrm{OC}$ to $\mathrm{NC}$ women during the follicular phase and luteal phase, has been associated with effects on emotion perception and processing. The authors hypothesized that thicker cortical volume in NC women compared to women taking OCs was driven by the follicular phase rather than the luteal phase [88]. In addition, grey matter changes were specifically seen in sex dimorphic

Neuroendocrinology 2021;111:183-206

197 
areas [87, 89]. When looking at possible underlying mechanisms at the microstructural level, a higher apparent diffusion coefficient of the hypothalamus was found in the pill-free week of women taking OCs [93]. Even though the exact mechanism remains speculative, these transitory changes in neurons or glia cells may finally lead to the activation of the gonadal axes and thereby increase endogenous hormones [93]. Effects of OC intake are quite inhomogeneous when evaluating different behavioural outcomes such as changes in mood with either positive or negative effects $[8,132]$ and either an increase or decrease in performance for specific cognitive tasks on cognition (for review please see $[28,133]$ ). Different types of OCs and use of progestines make comparisons between these studies and participants even more problematic. An underlying reason for behavioural differences may be the suppression of endogenous sex hormones in OC intake [134]. In animal studies and humans an impairment in fear extinction with higher skin conduction in women taking OCs could be observed, which may be specifically related to the suppression of estradiol levels [134]. Also partner preferences and social reward mechanisms seem to be altered due to a decrease in oxytocin sensitivity caused by estradiol suppression [131, 135]. With regard to emotional experiences, women taking OCs seem to experience blunted positive affect compared to NC women [136] and made more errors in terms of facial emotion recognition for anger, sadness and disgust ([137], for review please see [138]). Moreover, some women suffering from premenstrual mood swings may show an improvement in mood with OC intake [139]. Overall, women seem to report more anxiety, irritability or mood swings as well as higher intake of antidepressant medication when using OCs especially in women with previous depressive symptoms (for review please see [140]). In 2 randomized control trials including a placebo condition, women taking OCs reported a decrease in well-being, higher negative affect and a small effect on overall mood [138, 141]. This makes informing women about possible mood-related side effects of OC intake crucial and should be discussed openly. Data on other hormonal contraceptives such as intrauterine devices is missing entirely.

\section{Pregnancy and Postpartum Period}

Women during pregnancy showed decreases in grey matter for the anterior and posterior midline structures, bilateral prefrontal cortex, bilateral temporal cortex and right ventral striatum $[101,103,104]$. As areas also overlap with the Theory of Mind Network, this may support the assumption of an adaptive process preparing the mother for the nurturing role of looking after a baby [102]. In accordance with structural changes, behaviourally an increased sensitivity of the emotion processing system was reported which showed a correlation with rising sex hormones over the course of pregnancy [142, 143]. Thereby, women showed higher accuracy to encode emotional expressions that signal harm or threat and negative emotions [142] and showed better results for recognizing male faces [143]. In the postpartum period, an increase in grey matter including the prefrontal and parietal cortex as well as midbrain areas were reported [106]. Using the BrainAGE approach, brains of women were on average 5 years younger [105]. Moreover, decreased startle response modulation was observed after the presentation of emotional stimuli [144]. Women reported higher arousal and extremer valence ratings for both negative and positive stimuli. Reductions in ventral striatum volume across pregnancy were associated with higher activation of the reward circuit when being exposed to pictures of their offspring [103].

\section{Menopause}

Studies investigating the effects of MHT on GMV in postmenopausal women do not provide unanimous findings. Efforts to explain such differences exist but due to the limited number of studies can only be considered speculative at the moment. As suggested by Wnuk et al. [145] the diverse results that have appeared in previous studies on the impact of MHT on brain volume may be due to several moderating factors. One of these could be the length of MHT treatment. In this regard, Lord et al. [111] showed that within the group of currently treated women the total hippocampal volume was negatively associated with length of MHT duration suggesting smaller hippocampal volumes with longer MHT treatment. Similarly, Erickson et al. [146] found smaller grey matter volumes in areas including bilateral prefrontal cortex in women using MHT for longer periods. Another moderating factor could be the time between onset of menopause and the initiation of hormone therapy. Here, Erickson et al. [117] report that the more time has elapsed between the onset of menopause and hormone treatment, the smaller bilateral hippocampal volumes are. This effect was even observed when controlling for confounders like age and years of education, type of hormonal replacement, duration of therapy and age at menopause. Further dividing MHT users, regarding time of treatment, also showed that MHT was associated with increases on bilateral hippocampus volumes when hormone treatment was 
initiated within 1 year after onset of menopause. These observations are often described as a "critical-window" [147]. On a behavioural level, however, recent studies investigating the impact of MHT during early menopause did not show beneficial cognitive effects of MHT compared to placebo $[148,149]$, giving no support for such a critical-window-effect on cognitive aspects.

Thus, at the moment claims about the neuroprotective role of MHT appear to be more complex than a simple answer to a "yes-no" question. The comparability of studies is furthermore complicated by the heterogeneity of samples regarding age, mental health, length of MHTuse, healthy-user bias and kind of medication (Table 4). An association between MHT and better overall cognitive abilities in women such as working and spatial memory, attention as well as performance in verbal tasks has been reported and a reduced risk of developing dementia was found in some studies [150-153]. While in others, such as the Women's Health Initiative Memory Study, an adverse effect on cognition, which was greater among women with lower cognitive functioning at initiation of treatment was reported $[154,155]$. Some studies reported diminished verbal memory and spatial ability and reduced hippocampal and total brain volume [156, 157]. Again, others did not find a coupling of spatial memory performance and brain volumes $[146,148]$. Timing of menopause onset seems to play an important role, for example, experiencing premature menopause (before the age of 40) has been associated with poorer post-menopausal cognitive functioning and increased mortality rates [158]. This is in line with findings of decreases in brain areas important for memory and overall cognitive abilities such as the hippocampus and parietal cortex $[111,108]$. One reason for these divergent findings may also be the difference in age groups investigated (women of 50-60 years vs. women older than 70 years), as consequently, age-related declines and hormonal influences become difficult to disentangle. Interestingly, studies investigating the influence of regular exercise on brain structure in the older population often find increases in similar areas as with MHT, for example, the hippocampus [159]. One possible reason for this may be the increase of the brain-derived neurotrophic factor (BDNF) that often increases 2- to threefold during exercise [160]. Thereby, BDNF seems to increase synaptic plasticity, increasing effects of long-term potentiation [161]. In addition, there may be an interaction of estradiol receptors with BDNF [162]. In animal studies, previously ovariectomized young mice and rats that subsequently received MHT, showed increases of BDNF in several brain regions such as the hippocampus $[163,164]$

Shaping of the Female Brain by Sex Hormones amygdala [165] and overall cortex [163]. Moreover, kisspeptin seems to play an important role in the menopausal transition period [166, 167]. Using postmortem brain tissue, Rometo et al. [166] reported that kisspeptin 1 cells were predominantely located in the infundibular nucleus of the hypothalamus and that there was a significant increase in the size of neurons expressing KiSS-1 mRNA and the number of labelled cells and autoradiographic grains per neuron in tissue from postmenopausal compared to premenopausal women. Similar to postmenopausal women, ovariectomy induced neuronal hypertrophy and increased KiSS-1 gene expression in the infundibular nucleus of cynomolgus monkeys. However, oestrogen replacement markedly reduced KiSS-1 gene expression these in ovariectomized monkeys. Thus, these findings strongly point to the involvement of kisspeptin neurons in mediating the negative feedback of estradiol. Similarly, an immunocytochemical study revealed less and differently distributed and kisspeptin fibres and soma in men [167]. A proximity to GnRH bodies and dendrites was additionally confirmed.

\section{General Discussion}

Underlying mechanisms for structural changes may be diverse and can also be contradictory when comparing different hormones and their actions. At a structural and molecular level, estradiol has neurotrophic and neuroprotective effects on the brain by inducing synaptogenesis and an increase in spine density [168]. However, at the same time, it can initiate opposing effects such as cell apoptosis, that is, during developmental phases such as puberty [169]. Due to its action via classical gene transcription and membrane receptors it has the ability to influence cell functioning within several minutes or initiate longer lasting transcription of genes via genomic actions. Building upon results of neuronal growth and cognitive performance such as memory either a linear or an inverted U-shape model of estradiol action has been proposed $[170,171]$. One explanation for the incongruence in findings may be that only at supraphysiological levels the expression of ER alpha decreases which is not investigated in most studies [171]. Moreover, there seems to be a positive relationship between cerebral and central venous estradiol levels [172]. However, so far, the exact relationship is not fully clarified. In addition, estradiol is synthesized from testosterone via the P-450 aromatase in presynaptic terminals and converted through $\mathrm{Ca}^{2+}$ dependent phosphorylation [173]. Progesterone, however, 
may have opposing effects to estradiol such as inhibiting neural activity in specific subregions of the hippocampus, that is, CA1 and the dental gyrus [168]. In animal studies, high levels of progesterone led to a decrease in spine density and volume such as from proestrus to oestrus [174]. These effects were also observed during periods when both estradiol and progesterone were high, as is the case during the ML phase and pregnancy [104, 175]. Precursors of progesterone, for example, pregnanolone and allopregnanolone, are also directly synthesized in the brain from cholesterone by astrocytes and oligodendrocites [19]. In recent years the influence of kisspeptin on sex hormones has been further investigated. It not only seems to act as a gate keeper for the initiation of puberty, but is also involved in reproduction and reaches high levels during pregnancy due to being expressed by the placenta (for review please see [176]). Administration of kisspeptin in healthy women led to increases in gonadotropin levels especially during the preovulatory phase of the menstrual cycle [177]. Significantly higher levels of kisspeptin have also been found in the infundibular nucleus of postmenopausal women in a postmortem study and in ovariectomized cynomolgus monkeys [166]. Thereby, kisspeptin appears to be involved in 2 opposing mechanisms: the negative sex steroid feedback loop, by downregulating oestrogen and testosterone and the positive feedback loop by increasing gonadotropin levels during the prevovulatory phase (for review see [178]). Moreover, the influence of hormonal levels on other processes, for example, the neurotransmitter system or glia cells should not be underestimated (for review please see [23]). Metabolites of progesterone specifically bind to GABAA receptors and thereby can influence neuronal inhibition and induce anxiolytic and sedative effects (for review please see [175]). Similar effects could be observed for the testosterone-derived neurosteroid androstadienol that potentiated GABAA modulated outcomes [179]. Studies point towards an important role of disrupted neurosteroid functioning in postpartum depression, premenstrual symptoms and chronic stress $[176,180]$. For example, during pregnancy studies showed a decrease of inhibition through a decline of GABAAR $\delta$ and $\gamma^{2}$ that is reversed in the postpartum phase [17]. Moreover, in socially isolated mice a reduction of mRNA expression of $5 a-R I$, one of the enzymes important for the conversion of progesterone to allopregnanolone, was found [181]. This could have implications for the development of psychiatric symptoms, for example, anxiety and depression, as reduced GABA receptors and neurogenesis have been associated with heightened anxiety $[182,183]$. Sex and gender differences in mental disorders are a well-known and stable phenomenon in psychiatry [184]. Women are differently affected by mental and neurodegenerative disorders than men not only in terms of prevalence rates but also with regard to symptomatology, severity, course of illness, help-seeking behaviour, and treatment efficacy $[28,185,186]$. In particular, depression [187], eating disorders [188] and anxiety disorders [189] are more common in women than in men, starting at puberty. Importantly, their onset and relapse are often related to the physiological fluctuations of the menstrual cycle, as well as to reproductive events like pregnancy, postpartum and transition to menopause [42]. In addition, there is some evidence pointing towards microstructural changes in hormone-related structures such as the hypothalamus. Accordingly, a recent study reported a reduction of glutamate/glutamine (Glx) after food intake in patients with anorexia nervosa that was associated with a reduction of fibres in the arcuate nucleus and increased connectivity of the lateral hypothalamus [190]. The possible link between hormonal transition periods and female mental (Ill) health are not well understood, and surprisingly little research has been done to explain this association. However, understanding these associations by potentially looking at effects of sex hormones on brain morphology could give important insights into etiological and pathogenic mechanisms of these mental disorders, thereby most likely improving our therapeutic approaches for both women and men.

\section{Limitations and Suggestions for Future Studies}

Currently, differences in the reported study setups make an accurate comparison within different hormonal phases and across the reproductive lifespan troublesome. This becomes apparent especially during puberty when different age groups are investigated and varying methods/approaches for pubertal stage classification are used. One critical aspect is definitely the assessment of hormone levels, which is mandatory to draw accurate conclusions. The same criteria apply for assessing women in the transition period of menopause or the different menstrual cycle phases. As hormonal levels across the menstrual cycle show a great variety, within-subject and longitudinal designs may be of particular importance. Future studies should also aim for assessing behavioural measures to inform about the effects of potential grey matter changes. Most studies also rely on cross-sectional data that make it more difficult to exclude other possible in- 
fluencing factors such as life style and education, which may have an influence on the choice of MHT or OC use. Conducting more trials in a within-subject design by comparing pre- to post-treatment results may help to either control for these factors or highlight their importance. One limitation may be related to the neuroimaging method, as MR exclusion criteria lead to a selection bias and sample sizes are normally small due to several reasons among them high imaging costs. Consequently, this leads to underpowered studies that may not be able to detect true effects. Another restricting factor is that the exact relationship between peripheral hormonal levels and hormonal levels in the brain is not clarified yet [172]. Therefore, combining results from MRI studies with different methods such as positron emission tomography or epigenetic analyses would be helpful in order to develop a broader knowledge of hormonal mechanisms. Also, several studies failed to provide brain coordinates of significant findings and used different statistical contrasts and approaches (whole brain vs. region of interest analysis) making meta-analyses not possible at this moment. Overall, in order to make further conclusions more studies systematically investigating the role of hormonal fluctuations on brain structure are needed.

\section{Acknowledgement}

We thank Aiste Ambrase and Zoé Bürger for reviewing the manuscript.

\section{Disclosure Statement}

The authors have no conflicts of interest to declare.

\section{Funding Sources}

This study was funded by the Center for Integrative Neuroscience, Tuebingen (CIN, EXC307, MRTG Pregnancy and the Brain) and Deutsche Forschungsgemeinschaft (DFGDE2319/9-1).

\section{Author Contributions}

E.R. conducted the systematic literature search and wrote the manuscript. J.H. contributed to the literature search and manuscript. I.S.P. edited the manuscript and contributed with her knowledge. B.D. developed the idea for the paper, helped with editing and contributed with her knowledge.

\section{References}

1 Kolb B, Gibb R. Searching for the principles of brain plasticity and behavior. Cortex. 2014 Sep;58:251-60.

2 Phoenix CH, Goy RW, Gerall AA, Young WC. Organizing action of prenatally administered testosterone propionate on the tissues mediating mating behavior in the female guinea pig. Endocrinology. 1959 Sep;65(3): 369-82.

3 Arnold AP. The organizational-activational hypothesis as the foundation for a unified theory of sexual differentiation of all mammalian tissues. Horm Behav. 2009 May;55(5):570-8.

4 Juraska JM, Sisk CL, DonCarlos LL. Sexual differentiation of the adolescent rodent brain: hormonal influences and developmental mechanisms. Horm Behav. 2013 Jul;64(2):203-10.

5 Shaw P, Kabani NJ, Lerch JP, Eckstrand K, Lenroot R, Gogtay N, et al. Neurodevelopmental trajectories of the human cerebral cortex. J Neurosci. 2008 Apr;28(14):3586-94.

6 Sisk CL, Foster DL. The neural basis of puberty and adolescence. Nat Neurosci. 2004 Oct; 7(10):1040-7.

7 Toffoletto S, Lanzenberger R, Gingnell M, Sundström-Poromaa I, Comasco E. Emotional and cognitive functional imaging of estrogen and progesterone effects in the female human brain: a systematic review. Psychoneuroendocrinology. 2014 Dec;50:28-52.
8 Lisofsky N, Riediger M, Gallinat J, Lindenberger U, Kühn S. Hormonal contraceptive use is associated with neural and affective changes in healthy young women. Neuroimage. 2016 Jul; 134:597-606.

9 Comasco E, Sundström-Poromaa I. Neuroimaging the menstrual cycle and premenstrual dysphoric disorder. Curr Psychiatry Rep. 2015 Oct; 17(10):77.

10 Haraguchi S, Sasahara K, Shikimi H, Honda S, Harada N, Tsutsui K. Estradiol promotes purkinje dendritic growth, spinogenesis, and synaptogenesis during neonatal life by inducing the expression of BDNF. Cerebellum. 2012 Jun;11(2):416-7.

11 Cooke BM, Woolley CS. Gonadal hormone modulation of dendrites in the mammalian CNS. J Neurobiol. 2005 Jul;64(1):34-46.

12 Garcia-Segura LM, Melcangi RC. Steroids and glial cell function. Glia. 2006 Nov;54(6): 485-98.

13 Kawata M, Nishi M, Matsuda K, Sakamoto H, Kaku N, Masugi-Tokita M, et al. Steroid receptor signalling in the brain-lessons learned from molecular imaging. J Neuroendocrinol. 2008 Jun;20(6):673-6.

14 Zheng P. Neuroactive steroid regulation of neurotransmitter release in the CNS: action, mechanism and possible significance. Prog Neurobiol. 2009 Oct;89(2):134-52.
15 Singh M, Su C. Progesterone and neuroprotection. Horm Behav. 2013 Feb;63(2):284-90.

16 Moraga-Amaro R, van Waarde A, Doorduin J, de Vries EF. Sex steroid hormones and brain function: PET imaging as a tool for research. J Neuroendocrinol. 2018 Feb;30(2):e12565.

17 Osterlund MK, Gustafsson JA, Keller E, Hurd YL. Estrogen receptor beta (ERbeta) messenger ribonucleic acid (mRNA) expression within the human forebrain: distinct distribution pattern to ERalpha mRNA. J Clin Endocrinol Metab. 2000 Oct;85(10):3840-6.

18 Kato J, Hirata S, Nozawa A, Yamada-Mouri $\mathrm{N}$. Gene expression of progesterone receptor isoforms in the rat brain. Horm Behav. 1994 Dec;28(4):454-63.

19 Genazzani A, Stomati M, Morittu A, Bernardi F, Monteleone P, Casarosa E, et al. Progesterone, progestagens and the central nervous system. Hum Reprod. 2000 Jun;15 Suppl 1:14-27.

20 Ibanez C, Shields SA, El-Etr M, Leonelli E, Magnaghi V, Li WW, et al. Steroids and the reversal of age-associated changes in myelination and remyelination. Prog Neurobiol. 2003 Sep;71(1):49-56.

21 Cervantes M, González-Vidal MD, Ruelas R, Escobar A, Moralí G. Neuroprotective effects of progesterone on damage elicited by acute global cerebral ischemia in neurons of the caudate nucleus. Arch Med Res. 2002 Jan-Feb;33(1):6-14. 
22 Bixo M, Andersson A, Winblad B, Purdy RH, Bäckström T. Progesterone, 5a-pregnane3,20-dione and 3a-hydroxy-5a-pregnane-20one in specific regions of the human female brain in different endocrine states. Brain Res. 1997 Aug;764(1-2):173-8.

23 Barth C, Villringer A, Sacher J. Sex hormones affect neurotransmitters and shape the adult female brain during hormonal transition periods. Front Neurosci. 2015 Feb;9:37.

24 Gruber CJ, Tschugguel W, Schneeberger C, Huber JC. Production and actions of estrogens. N Engl J Med. 2002 Jan;346(5):340-52.

25 Cunningham RL, Lumia AR, McGinnis MY. Androgen receptors, sex behavior, and aggression. Neuroendocrinology. 2012;96(2): 131-40.

26 Österlund MK, Keller E, Hurd YL. The human forebrain has discrete estrogen receptor a messenger RNA expression: high levels in the amygdaloid complex. Neuroscience. 2000;95(2):333-42.

27 Thomas MP, Potter BV. The structural biology of oestrogen metabolism. J Steroid Biochem Mol Biol. 2013 Sep;137:27-49.

28 Gogos A, Wu YC, Williams AS, Byrne LK. The effects of ethinylestradiol and progestins ("the pill") on cognitive function in premenopausal women. Neurochem Res. 2014 Dec;39(12):2288-300.

29 Jeyakumar M, Carlson KE, Gunther JR, Katzenellenbogen JA. Exploration of dimensions of estrogen potency: parsing ligand binding and coactivator binding affinities. J Biol Chem. 2011 Apr;286(15):12971-82.

30 Sun J, Walker AJ, Dean B, van den Buuse M, Gogos A. Progesterone: the neglected hormone in schizophrenia? A focus on progesterone-dopamine interactions. Psychoneuroendocrinology. 2016 Dec;74:126-40.

31 Greenblatt RB, Oettinger M, Bohler CS. Estrogen-androgen levels in aging men and women: therapeutic considerations. J Am Geriatr Soc. 1976 Apr;24(4):173-8.

32 Orwoll E, Lambert LC, Marshall LM, Phipps $\mathrm{K}$, Blank J, Barrett-Connor E, et al. Testosterone and estradiol among older men. Clin Endocrinol Metab. 2006 Apr;91(4): 1336-44.

33 Stricker R, Eberhart R, Chevailler MC, Quinn FA, Bischof P, Stricker R. Establishment of detailed reference values for luteinizing hormone, follicle stimulating hormone, estradiol, and progesterone during different phases of the menstrual cycle on the Abbott ARCHITECT analyzer [CCLM]. Clin Chem Lab Med. 2006;44(7):883-7.

34 Sundström Poromaa I, Gingnell M. Menstrual cycle influence on cognitive function and emotion processing-from a reproductive perspective. Front Neurosci. 2014 Nov; 8:380.

35 Schock H, Zeleniuch-Jacquotte A, Lundin E, Grankvist K, Lakso HÅ, Idahl A, et al. Hormone concentrations throughout uncomplicated pregnancies: a longitudinal study. BMC Pregnancy Childbirth. 2016 Jul;16(1):146.
36 Burger HG, Dudley EC, Robertson DM, Dennerstein L. Hormonal changes in the menopause transition. Recent Prog Horm Res. 2002;57(1):257-75.

37 Oettel M, Mukhopadhyay AK. Progesterone: the forgotten hormone in men? Aging Male. 2004 Sep;7(3):236-57.

38 Mumford SL, Schisterman EF, Siega-Riz AM, Browne RW, Gaskins AJ, Trevisan M, et al. A longitudinal study of serum lipoproteins in relation to endogenous reproductive hormones during the menstrual cycle: findings from the BioCycle study. J Clin Endocrinol Metab. 2010 Sep;95(9):E80-5.

39 Byrns MC. Regulation of progesterone signaling during pregnancy: implications for the use of progestins for the prevention of preterm birth. J Steroid Biochem Mol Biol. 2014 Jan;139:173-81.

40 Santoro N. The menopausal transition. Am J Med. 2005 Dec;118(Suppl 12B):8-13.

41 Marsh WK, Ketter TA, Crawford SL, Johnson JV, Kroll-Desrosiers AR, Rothschild AJ. Progression of female reproductive stages associated with bipolar illness exacerbation. Bipolar Disord. 2012 Aug;14(5):515-26.

42 Soares CN, Zitek B. Reproductive hormone sensitivity and risk for depression across the female life cycle: a continuum of vulnerability? J Psychiatry Neurosci. 2008 Jul;33(4):33143.

43 Albert KM, Newhouse PA. Estrogen, stress, and depression: cognitive and biological interactions. Annu Rev Clin Psychol. 2019 May; 15(1):399-423.

44 Lokuge S, Frey BN, Foster JA, Soares CN, Steiner M. Depression in women: windows of vulnerability and new insights into the link between estrogen and serotonin. J Clin Psychiatry. 2011 Nov;72(11):e1563-9.

45 Sundström I, Bäckström T, Wang M, Olsson T, Seippel L, Bixo M. Premenstrual syndrome, neuroactive steroids and the brain. Gynecol Endocrinol. 1999 Jun;13(3):206-20.

46 Segebladh B, Borgström A, Odlind V, Bixo M, Sundström-Poromaa I. Prevalence of psychiatric disorders and premenstrual dysphoric symptoms in patients with experience of adverse mood during treatment with combined oral contraceptives. Contraception. 2009 Jan; 79(1):50-5

47 Bäckström T, Bixo M, Johansson M, Nyberg S, Ossewaarde L, Ragagnin G, et al. Allopregnanolone and mood disorders. Prog Neurobiol. 2014 Feb;113:88-94.

48 McEvoy K, Osborne LM. Allopregnanolone and reproductive psychiatry: an overview. Int Rev Psychiatry. 2019 May;31(3):237-44.

49 Catenaccio E, Mu W, Lipton ML. Estrogenand progesterone-mediated structural neuroplasticity in women: evidence from neuroimaging. Brain Struct Funct. 2016 Nov;221(8): 3845-67.

50 Walker EF, Sabuwalla Z, Huot R. Pubertal neuromaturation, stress sensitivity, and psychopathology. Dev Psychopathol. 2004;16(4): 807-24.
51 Ojeda SR, Prevot V, Heger S, Lomniczi A, Dziedzic B, Mungenast A. The neurobiology of female puberty. Horm Res. 2003;60 Suppl 3:15-20.

52 Sisk CL, Zehr JL. Pubertal hormones organize the adolescent brain and behavior. Front Neuroendocrinol. 2005 Oct-Dec;26(3-4): 163-74.

53 Pineda R, Garcia-Galiano D, Roseweir A, Romero M, Sanchez-Garrido MA, Ruiz-Pino F, et al. Critical roles of kisspeptins in female puberty and preovulatory gonadotropin surges as revealed by a novel antagonist. Endocrinology. 2010 Feb;151(2):722-30.

54 d'Anglemont de Tassigny X, Fagg LA, Carlton $\mathrm{MB}$, Colledge WH. Kisspeptin can stimulate gonadotropin-releasing hormone $(\mathrm{GnRH})$ release by a direct action at GnRH nerve terminals. Endocrinology. 2008 Aug; 149(8): 3926-32.

55 Zuloaga DG, Puts DA, Jordan CL, Breedlove $\mathrm{SM}$. The role of androgen receptors in the masculinization of brain and behavior: what we've learned from the testicular feminization mutation. Horm Behav. 2008 May;53(5):61326.

56 Parent AS, Teilmann G, Juul A, Skakkebaek NE, Toppari J, Bourguignon JP. The timing of normal puberty and the age limits of sexual precocity: variations around the world, secular trends, and changes after migration. Endocr Rev. 2003 Oct;24(5):668-93.

57 Tanner JM, Davies PS. Clinical longitudinal standards for height and height velocity for North American children. J Pediatr. 1985 Sep; 107(3):317-29.

58 Petersen AC, Crockett L, Richards M, Boxer A. A self-report measure of pubertal status: Reliability, validity, and initial norms. J Youth Adolesc. 1988 Apr;17(2):117-33.

59 Marshall WA, Tanner JM. Puberty. Postnatal growth neurobiology. Springer; 1986. pp. 171-209.

60 Giedd JN, Blumenthal J, Jeffries NO, Castellanos FX, Liu H, Zijdenbos A, et al. Brain development during childhood and adolescence: a longitudinal MRI study. Nat Neurosci. 1999 Oct;2(10):861-3.

61 Gogtay N, Giedd JN, Lusk L, Hayashi KM, Greenstein D, Vaituzis AC, et al. Dynamic mapping of human cortical development during childhood through early adulthood. Proc Natl Acad Sci USA. 2004 May;101(21):8174-9.

62 Lebel C, Beaulieu C. Longitudinal development of human brain wiring continues from childhood into adulthood. J Neurosci. 2011 Jul;31(30):10937-47.

63 Lenroot RK, Gogtay N, Greenstein DK, Wells EM, Wallace GL, Clasen LS, et al. Sexual dimorphism of brain developmental trajectories during childhood and adolescence. Neuroimage. 2007 Jul;36(4):1065-73.

64 Blakemore SJ, Choudhury S. Development of the adolescent brain: implications for executive function and social cognition. J Child Psychol Psychiatry. 2006 Mar-Apr;47(3-4): 296-312. 
65 Casey BJ, Jones RM, Levita L, Libby V, Pattwell SS, Ruberry EJ, et al. The storm and stress of adolescence: insights from human imaging and mouse genetics. Dev Psychobiol. 2010 Apr;52(3):225-35.

66 Sahlberg BL, Landgren BM, Axelson M. Metabolic profiles of endogenous and ethynyl steroids in plasma and urine from women during administration of oral contraceptives. J Steroid Biochem. 1987 May;26(5):609-17.

67 Hendrick V, Altshuler LL, Suri R. Hormonal changes in the postpartum and implications for postpartum depression. Psychosomatics. 1998 Mar-Apr;39(2):93-101.

68 Morrison JH, Brinton RD, Schmidt PJ, Gore AC. Estrogen, menopause, and the aging brain: how basic neuroscience can inform hormone therapy in women. J Neurosci. 2006 Oct;26(41):10332-48.

69 Sievert LL. Menopause across cultures: clinical considerations. Menopause. 2014 Apr; 21(4):421-3.

70 Baker FC, de Zambotti M, Colrain IM, Bei B. Sleep problems during the menopausal transition: prevalence, impact, and management challenges. Nat Sci Sleep. 2018 Feb;10:73-95.

71 Marjoribanks J, Farquhar CM, Roberts H, Lethaby A. Cochrane corner: long-term hormone therapy for perimenopausal and postmenopausal women. Heart. 2018 Jan;104(2): 93-5.

72 Bramen JE, Hranilovich JA, Dahl RE, Forbes EE, Chen J, Toga AW, et al. Puberty influences medial temporal lobe and cortical gray matter maturation differently in boys than girls matched for sexual maturity. Cereb Cortex. 2011 Mar;21(3):636-46.

73 Peper JS, Brouwer RM, Schnack HG, van Baal GC, van Leeuwen M, van den Berg SM, et al. Cerebral white matter in early puberty is associated with luteinizing hormone concentrations. Psychoneuroendocrinology. 2008 Aug; 33(7):909-15.

74 Herting MM, Gautam P, Spielberg JM, Dahl $\mathrm{RE}$, Sowell ER. A longitudinal study: changes in cortical thickness and surface area during pubertal maturation. PLoS One. 2015 Mar; 10(3):e0119774.

75 Peper JS, Brouwer RM, Schnack HG, van Baal GC, van Leeuwen M, van den Berg SM, et al. Sex steroids and brain structure in pubertal boys and girls. Psychoneuroendocrinology. 2009 Apr;34(3):332-42.

76 Koolschijn PC, Peper JS, Crone EA. The influence of sex steroids on structural brain maturation in adolescence. PLoS One. 2014 Jan; 9(1):e83929.

77 Neufang S, Specht K, Hausmann M, Güntürkün $\mathrm{O}$, Herpertz-Dahlmann B, Fink GR, et al. Sex differences and the impact of steroid hormones on the developing human brain. Cereb Cortex. 2009 Feb;19(2):464-73.

78 Perrin JS, Hervé PY, Leonard G, Perron M, Pike GB, Pitiot A, et al. Growth of white matter in the adolescent brain: role of testosterone and androgen receptor. J Neurosci. 2008 Sep; 28(38):9519-24.
79 Paus T, Nawaz-Khan I, Leonard G, Perron M, Pike GB, Pitiot A, et al. Sexual dimorphism in the adolescent brain: role of testosterone and androgen receptor in global and local volumes of grey and white matter. Horm Behav. 2010 Jan;57(1):63-75.

80 Herting MM, Gautam P, Spielberg JM, Kan E, Dahl RE, Sowell ER. The role of testosterone and estradiol in brain volume changes across adolescence: a longitudinal structural MRI study. Hum Brain Mapp. 2014 Nov;35(11): 5633-45.

81 Herting MM, Maxwell EC, Irvine C, Nagel BJ. The impact of sex, puberty, and hormones on white matter microstructure in adolescents. Cereb Cortex. 2012 Sep;22(9): 1979-92.

82 Hagemann G, Ugur T, Schleussner E, Mentzel HJ, Fitzek C, Witte OW, et al. Changes in brain size during the menstrual cycle. PLoS One. 2011 Feb;6(2):e14655.

83 Lisofsky N, Mårtensson J, Eckert A, Lindenberger U, Gallinat J, Kühn S. Hippocampal volume and functional connectivity changes during the female menstrual cycle. Neuroimage. 2015 Sep;118:154-62.

84 Protopopescu X, Butler T, Pan H, Root J, Altemus M, Polanecsky M, et al. Hippocampal structural changes across the menstrual cycle. Hippocampus. 2008;18(10):985-8.

85 Ossewaarde L, van Wingen GA, Rijpkema M, Bäckström T, Hermans EJ, Fernández G. Menstrual cycle-related changes in amygdala morphology are associated with changes in stress sensitivity. Hum Brain Mapp. 2013 May;34(5):1187-93.

86 De Bondt T, Pullens P, Van Hecke W, Jacquemyn Y, Parizel PM. Reproducibility of hormone-driven regional grey matter volume changes in women using SPM8 and SPM12. Brain Struct Funct. 2016 Dec;221(9):463141.

87 Pletzer B, Kronbichler M, Aichhorn M, Bergmann J, Ladurner G, Kerschbaum HH. Menstrual cycle and hormonal contraceptive use modulate human brain structure. Brain Res. 2010 Aug; 1348:55-62.

88 Petersen N, Touroutoglou A, Andreano JM, Cahill L. Oral contraceptive pill use is associated with localized decreases in cortical thickness. Hum Brain Mapp. 2015 Jul;36(7):264454.

89 Pletzer B, Kronbichler M, Kerschbaum H. Differential effects of androgenic and anti-androgenic progestins on fusiform and frontal gray matter volume and face recognition performance. Brain Res. 2015 Jan;1596:108-15.

90 De Bondt T, Jacquemyn Y, Van Hecke W, Sijbers J, Sunaert S, Parizel PM. Regional gray matter volume differences and sex-hormone correlations as a function of menstrual cycle phase and hormonal contraceptives use. Brain Res. 2013 Sep;1530:22-31.

91 Barth C, Steele CJ, Mueller K, Rekkas VP, Arélin K, Pampel A, et al. In-vivo Dynamics of the Human Hippocampus across the Menstrual Cycle. Sci Rep. 2016 Oct;6(1):32833.
92 De Bondt T, Van Hecke W, Veraart J, Leemans A, Sijbers J, Sunaert S, et al. Does the use of hormonal contraceptives cause microstructural changes in cerebral white matter? Preliminary results of a DTI and tractography study. Eur Radiol. 2013 Jan;23(1): 57-64.

93 Baroncini M, Jissendi P, Catteau-Jonard S, Dewailly D, Pruvo JP, Francke JP, et al. Sex steroid hormones-related structural plasticity in the human hypothalamus. Neuroimage. 2010 Apr;50(2):428-33.

94 Witte AV, Savli M, Holik A, Kasper S, Lanzenberger R. Regional sex differences in grey matter volume are associated with sex hormones in the young adult human brain. Neuroimage. 2010 Jan;49(2):120512.

95 Franke K, Hagemann G, Schleussner E, Gaser C. Changes of individual BrainAGE during the course of the menstrual cycle. Neuroimage. 2015 Jul;115:1-6.

96 Franke K, Ziegler G, Klöppel S, Gaser C; Alzheimer's Disease Neuroimaging Initiative. Estimating the age of healthy subjects from T1-weighted MRI scans using kernel methods: exploring the influence of various parameters. Neuroimage. 2010 Apr;50(3):88392.

97 Franke K, Luders E, May A, Wilke M, Gaser C. Brain maturation: predicting individual BrainAGE in children and adolescents using structural MRI. Neuroimage. 2012 Nov; 63(3):1305-12.

98 Gonzalez JG, Elizondo G, Saldivar D, Nanez H, Todd LE, Villarreal JZ. Pituitary gland growth during normal pregnancy: an in vivo study using magnetic resonance imaging. Am J Med. 1988 Aug;85(2):21720.

99 Elster AD, Sanders TG, Vines FS, Chen MY. Size and shape of the pituitary gland during pregnancy and post partum: measurement with MR imaging. Radiology. 1991 Nov; 181(2):531-5.

100 Dinç H, Esen F, Demirci A, Sari A, Resit Gümele H. Pituitary dimensions and volume measurements in pregnancy and post partum. MR assessment. Acta Radiol. 1998 Jan;39(1):64-9.

101 Hoekzema E, Barba-Müller E, Pozzobon C, Picado M, Lucco F, García-García D, et al. Pregnancy leads to long-lasting changes in human brain structure. Nat Neurosci. 2017 Feb;20(2):287-96.

102 Schurz M, Radua J, Aichhorn M, Richlan F, Perner J. Fractionating theory of mind: a meta-analysis of functional brain imaging studies. Neurosci Biobehav Rev. 2014 May; 42:9-34.

103 Hoekzema E, Tamnes CK, Berns P, BarbaMüller E, Pozzobon C, Picado M, et al. Becoming a mother entails anatomical changes in the ventral striatum of the human brain that facilitate its responsiveness to offspring cues. Psychoneuroendocrinology. $2020 \mathrm{Feb}$; 112:104507. 
104 Oatridge A, Holdcroft A, Saeed N, Hajnal JV, Puri BK, Fusi L, et al. Change in brain size during and after pregnancy: study in healthy women and women with preeclampsia. AJNR Am J Neuroradiol. 2002 Jan;23(1): 19-26.

105 Luders E, Gingnell M, Poromaa IS, Engman J, Kurth F, Gaser C. Potential brain age reversal after pregnancy: younger brains at 4-6 weeks postpartum. Neuroscience. 2018 Aug; 386:309-14.

106 Kim P, Leckman JF, Mayes LC, Feldman R, Wang X, Swain JE. The plasticity of human maternal brain: longitudinal changes in brain anatomy during the early postpartum period. Behav Neurosci. 2010 Oct;124(5): 695-700.

107 Albert K, Hiscox J, Boyd B, Dumas J, Taylor W, Newhouse P. Estrogen enhances hippocampal gray-matter volume in young and older postmenopausal women: a prospective dose-response study. Neurobiol Aging. 2017 Aug;56:1-6.

108 Eberling JL, Wu C, Haan MN, Mungas D, Buonocore M, Jagust WJ. Preliminary evidence that estrogen protects against age-related hippocampal atrophy. Neurobiol Aging. 2003 Sep;24(5):725-32.

109 Erickson KI, Colcombe SJ, Raz N, Korol DL, Scalf P, Webb A, et al. Selective sparing of brain tissue in postmenopausal women receiving hormone replacement therapy. Neurobiol Aging. 2005 Aug-Sep;26(8):1205-13.

110 Boccardi M, Ghidoni R, Govoni S, Testa C, Benussi L, Bonetti M, et al. Effects of hormone therapy on brain morphology of healthy postmenopausal women: a Voxelbased morphometry study. Menopause. 2006 Jul-Aug;13(4):584-91.

111 Lord C, Buss C, Lupien SJ, Pruessner JC. Hippocampal volumes are larger in postmenopausal women using estrogen therapy compared to past users, never users and men: a possible window of opportunity effect. Neurobiol Aging. 2008 Jan;29(1):95-101.

112 Eberling JL, Wu C, Tong-Turnbeaugh R, Jagust WJ. Estrogen- and tamoxifen-associated effects on brain structure and function. Neuroimage. 2004 Jan;21(1):364-71.

113 Greenberg DL, Payne ME, MacFall JR, Provenzale JM, Steffens DC, Krishnan RR. Differences in brain volumes among males and female hormone-therapy users and nonusers. Psychiatry Res. 2006 Oct;147(23):127-34.

114 Resnick SM, Espeland MA, Jaramillo SA, Hirsch C, Stefanick ML, Murray AM, et al. Postmenopausal hormone therapy and regional brain volumes: the WHIMS-MRI Study. Neurology. 2009 Jan;72(2):135-42.

115 Zhang T, Casanova R, Resnick SM, Manson JE, Baker LD, Padual CB, et al. Effects of hormone therapy on brain volumes changes of postmenopausal women revealed by optimally-discriminative voxel-based morphometry. PLoS One. 2016 Mar; 11(3):e0150834.
116 Kantarci K, Tosakulwong N, Lesnick TG, Zuk SM, Gunter JL, Gleason CE, et al. Effects of hormone therapy on brain structure: $\mathrm{A}$ randomized controlled trial. Neurology. 2016 Aug;87(9):887-96.

117 Erickson KI, Voss MW, Prakash RS, Chaddock L, Kramer AF. A cross-sectional study of hormone treatment and hippocampal volume in postmenopausal women: evidence for a limited window of opportunity. Neuropsychology. 2010 Jan;24(1):68-76.

118 Kim GW, Park K, Jeong GW. Effects of Sex Hormones and Age on Brain Volume in Post-Menopausal Women. J Sex Med. 2018 May;15(5):662-70.

119 Giorgio A, Watkins KE, Chadwick M, James S, Winmill L, Douaud G, et al. Longitudinal changes in grey and white matter during adolescence. Neuroimage. 2010 Jan;49(1):94-103.

120 Paus T. Mapping brain maturation and cognitive development during adolescence. Trends Cogn Sci. 2005 Feb;9(2):60-8.

121 Miller DJ, Duka T, Stimpson CD, Schapiro SJ, Baze WB, McArthur MJ, et al. Prolonged myelination in human neocortical evolution. Proc Natl Acad Sci USA. 2012 Oct; 109(41):16480-5.

122 Forbes EE, Dahl RE. Pubertal development and behavior: hormonal activation of social and motivational tendencies. Brain Cogn. 2010 Feb;72(1):66-72.

123 Romer D. Adolescent risk taking, impulsivity, and brain development: implications for prevention. Dev Psychobiol. 2010 Apr; 52(3):263-76.

124 Whelan R, Conrod PJ, Poline JB, Lourdusamy A, Banaschewski T, Barker GJ, et al.; IMAGEN Consortium. Adolescent impulsivity phenotypes characterized by distinct brain networks. Nat Neurosci. 2012 Jun;15(6):920-5.

125 McClure J, Podos J, Richardson HN. Isolating the delay component of impulsive choice in adolescent rats. Front Integr Nuerosci. 2014 Jan;8:3.

126 Guapo VG, Graeff FG, Zani AC, Labate CM, dos Reis RM, Del-Ben CM. Effects of sex hormonal levels and phases of the menstrual cycle in the processing of emotional faces. Psychoneuroendocrinology. 2009 Aug; 34(7):1087-94.

127 Derntl B, Kryspin-Exner I, Fernbach E, Moser E, Habel U. Emotion recognition accuracy in healthy young females is associated with cycle phase. Horm Behav. 2008 Jan; 53(1):90-5.

128 Derntl B, Windischberger C, Robinson S, Lamplmayr E, Kryspin-Exner I, Gur RC, et al. Facial emotion recognition and amygdala activation are associated with menstrual cycle phase. Psychoneuroendocrinology. 2008 Sep;33(8):1031-40.

129 Reed SC, Levin FR, Evans SM. Changes in mood, cognitive performance and appetite in the late luteal and follicular phases of the menstrual cycle in women with and without PMDD (premenstrual dysphoric disorder). Horm Behav. 2008 Jun;54(1):185-93.
130 Rubinow DR, Smith MJ, Schenkel LA Schmidt PJ, Dancer K. Facial emotion discrimination across the menstrual cycle in women with premenstrual dysphoric disorder (PMDD) and controls. J Affect Disord. 2007 Dec;104(1-3):37-44.

131 Montoya ER, Bos PA. How oral contraceptives impact social-emotional behavior and brain function. Trends Cogn Sci. 2017 Feb 21(2):125-36

132 Gingnell M, Engman J, Frick A, Moby L, Wikström J, Fredrikson M, et al. Oral contraceptive use changes brain activity and mood in women with previous negative affect on the pill-a double-blinded, placebocontrolled randomized trial of a levonorgestrel-containing combined oral contraceptive. Psychoneuroendocrinology. 2013 Jul; 38(7):1133-44

133 Warren AM, Gurvich C, Worsley R, Kulkarni J. A systematic review of the impact of oral contraceptives on cognition. Contraception. 2014 Aug;90(2):111-6.

134 Graham BM, Milad MR. Blockade of estrogen by hormonal contraceptives impairs fear extinction in female rats and women. Biol Psychiatry. 2013 Feb;73(4):371-8.

135 Scheele D, Plota J, Stoffel-Wagner B, Maier W, Hurlemann R. Hormonal contraceptives suppress oxytocin-induced brain reward responses to the partner's face. Soc Cogn Affect Neurosci. 2016 May;11(5):767-74.

136 Jarva JA, Oinonen KA. Do oral contraceptives act as mood stabilizers? Evidence of positive affect stabilization. Arch Women Ment Health. 2007;10(5):225-34.

137 Hamstra DA, De Rover M, De Rijk RH, Van der Does W. Oral contraceptives may alter the detection of emotions in facial expressions. Eur Neuropsychopharmacol. 2014 Nov;24(11):1855-9.

138 Lundin C, Danielsson KG, Bixo M, Moby L, Bengtsdotter H, Jawad I, et al. Combined oral contraceptive use is associated with both improvement and worsening of mood in the different phases of the treatment $c y$ cle-A double-blind, placebo-controlled randomized trial. Psychoneuroendocrinology. $2017 \mathrm{Feb} ; 76: 135-43$.

139 Joffe H, Cohen LS, Harlow BL. Impact of oral contraceptive pill use on premenstrual mood: predictors of improvement and deterioration. Am J Obstet Gynecol. 2003 Dec; 189(6):1523-30.

140 Lewis CA, Kimmig AS, Zsido RG, Jank A, Derntl B, Sacher J. Effects of Hormonal Contraceptives on Mood: A Focus on Emotion Recognition and Reactivity, Reward Processing, and Stress Response. Curr Psychiatry Rep. 2019 Nov;21(11):115.

141 Zethraeus N, Dreber A, Ranehill E, Blomberg L, Labrie F, von Schoultz B, et al. A firstchoice combined oral contraceptive influences general well-being in healthy women: a double-blind, randomized, placebo-controlled trial. Fertil Steril. 2017 May;107(5): $1238-45$. 
142 Pearson RM, Lightman SL, Evans J. Emotional sensitivity for motherhood: late pregnancy is associated with enhanced accuracy to encode emotional faces. Horm Behav. 2009 Nov;56(5):557-63.

143 Anderson MV, Rutherford MD. Recognition of novel faces after single exposure is enhanced during pregnancy. Evol Psychol. 2011 Feb;9(1):47-60.

144 Hellgren C, Bannbers E, Åkerud H, Risbrough V, Poromaa IS. Decreased startle modulation during anticipation in the postpartum period in comparison to late pregnancy. Arch Women Ment Health. 2012 Apr;15(2):87-94.

145 Wnuk A, Korol DL, Erickson KI. Estrogens, hormone therapy, and hippocampal volume in postmenopausal women. Maturitas. 2012 Nov;73(3):186-90.

146 Erickson KI, Colcombe SJ, Elavsky S, McAuley E, Korol DL, Scalf PE, et al. Interactive effects of fitness and hormone treatment on brain health in postmenopausal women. Neurobiol Aging. 2007 Feb;28(2):179-85.

147 Henderson VW, St John JA, Hodis HN, McCleary CA, Stanczyk FZ, Shoupe D, et al. Cognitive effects of estradiol after menopause: A randomized trial of the timing hypothesis. Neurology. 2016 Aug;87(7):699708.

148 Espeland MA, Shumaker SA, Leng I, Manson JE, Brown CM, LeBlanc ES, et al.; WHIMSY Study Group. Long-term effects on cognitive function of postmenopausal hormone therapy prescribed to women aged 50 to 55 years. JAMA Intern Med. 2013 Aug; 173(15):1429-36.

149 Gleason CE, Dowling NM, Wharton W, Manson JE, Miller VM, Atwood CS, et al. Effects of hormone therapy on cognition and mood in recently postmenopausal women: findings from the randomized, controlled KEEPS-Cognitive and Affective Study. PLoS Med. 2015 Jun;12(6):e1001833.

150 Keenan PA, Ezzat WH, Ginsburg K, Moore GJ. Prefrontal cortex as the site of estrogen's effect on cognition. Psychoneuroendocrinology. 2001 Aug;26(6):577-90.

151 Greendale GA, Huang MH, Wight RG, Seeman T, Luetters C, Avis NE, et al. Effects of the menopause transition and hormone use on cognitive performance in midlife women. Neurology. 2009 May;72(21):1850-7.

152 Dubal DB, Wilson ME, Wise PM. Estradiol: a protective and trophic factor in the brain. J Alzheimers Dis. 1999 Nov;1(4-5):265-74.

153 Maki PM. Verbal memory and menopause. Maturitas. 2015 Nov;82(3):288-90.

154 Espeland MA, Rapp SR, Shumaker SA, Brunner R, Manson JE, Sherwin BB, et al.; Women's Health Initiative Memory Study. Conjugated equine estrogens and global cognitive function in postmenopausal women: Women's Health Initiative Memory Study. JAMA. 2004 Jun;291(24):2959-68.

155 Shumaker SA, Legault C, Kuller L, Rapp SR, Thal L, Lane DS, et al.; Women's Health Ini- tiative Memory Study. Conjugated equine estrogens and incidence of probable dementia and mild cognitive impairment in postmenopausal women: Women's Health Initiative Memory Study. JAMA. 2004 Jun; 291(24):2947-58.

156 Coker LH, Espeland MA, Rapp SR, Legault C, Resnick SM, Hogan P, et al. Postmenopausal hormone therapy and cognitive outcomes: the Women's Health Initiative Memory Study (WHIMS). J Steroid Biochem Mol Biol. 2010 Feb;118(4-5):304-10.

157 Espeland MA, Tindle HA, Bushnell CA, Jaramillo SA, Kuller LH, Margolis KL, et al.; Women's Health Initiative Memory Study. Brain volumes, cognitive impairment, and conjugated equine estrogens. J Gerontol A Biol Sci Med Sci. 2009 Dec;64(12):1243-50.

158 Ryan J, Scali J, Carrière I, Amieva H, Rouaud $\mathrm{O}$, Berr $\mathrm{C}$, et al. Impact of a premature menopause on cognitive function in later life. BJOG. 2014 Dec;121(13):1729-39.

159 Erickson KI, Voss MW, Prakash RS, Basak C, Szabo A, Chaddock L, et al. Exercise training increases size of hippocampus and improves memory. Proc Natl Acad Sci USA. $2011 \mathrm{Feb} ; 108(7): 3017-22$.

160 Rasmussen P, Brassard P, Adser H, Pedersen MV, Leick L, Hart E, et al. Evidence for a release of brain-derived neurotrophic factor from the brain during exercise. Exp Physiol. 2009 Oct;94(10):1062-9.

161 Kuipers SD, Bramham CR. Brain-derived neurotrophic factor mechanisms and function in adult synaptic plasticity: new insights and implications for therapy. Curr Opin Drug Discov Devel. 2006 Sep;9(5):580-6.

162 Sohrabji F, Lewis DK. Estrogen-BDNF interactions: implications for neurodegenerative diseases. Front Neuroendocrinol. 2006 Dec;27(4):404-14.

163 Allen AL, McCarson KE. Estrogen increases nociception-evoked brain-derived neurotrophic factor gene expression in the female rat. Neuroendocrinology. 2005;81(3):193-9.

164 Scharfman HE, MacLusky NJ. Estrogengrowth factor interactions and their contributions to neurological disorders. Headache. 2008 Jul;48 Suppl 2:S77-89.

165 Zhou J, Zhang H, Cohen RS, Pandey SC. Effects of estrogen treatment on expression of brain-derived neurotrophic factor and cAMP response element-binding protein expression and phosphorylation in rat amygdaloid and hippocampal structures. Neuroendocrinology. 2005;81(5):294-310.

166 Rometo AM, Krajewski SJ, Voytko ML, Rance NE. Hypertrophy and increased kisspeptin gene expression in the hypothalamic infundibular nucleus of postmenopausal women and ovariectomized monkeys. J Clin Endocrinol Metab. 2007 Jul;92(7):2744-50.

167 Hrabovszky E, Ciofi P, Vida B, Horvath MC, Keller E, Caraty A, et al. The kisspeptin system of the human hypothalamus: sexual dimorphism and relationship with gonadotropin-releasing hormone and neurokinin $\mathrm{B}$ neurons. Eur J Neurosci. 2010 Jun;31(11): 1984-98.

168 Woolley CS, McEwen BS. Roles of estradiol and progesterone in regulation of hippocampal dendritic spine density during the estrous cycle in the rat. J Comp Neurol. 1993 Oct;336(2):293-306.

169 McCarthy MM. Estradiol and the developing brain. Physiol Rev. 2008 Jan;88(1):91124.

170 McLaughlin KJ, Bimonte-Nelson H, Neisewander JL, Conrad CD. Assessment of estradiol influence on spatial tasks and hippocampal CA1 spines: evidence that the duration of hormone deprivation after ovariectomy compromises $17 \beta$-estradiol effectiveness in altering CA1 spines. Horm Behav. 2008 Aug; 54(3):386-95.

171 Bayer J, Gläscher J, Finsterbusch J, Schulte $\mathrm{LH}$, Sommer T. Linear and inverted Ushaped dose-response functions describe estrogen effects on hippocampal activity in young women. Nat Commun. 2018 Mar; 9(1): 1220

172 Caruso D, Pesaresi M, Abbiati F, Calabrese D, Giatti S, Garcia-Segura LM, et al. Comparison of plasma and cerebrospinal fluid levels of neuroactive steroids with their brain, spinal cord and peripheral nerve levels in male and female rats. Psychoneuroendocrinology. 2013 Oct;38(10):2278-90.

173 Balthazart J, Baillien M, Ball GF. Rapid control of brain aromatase activity by glutamatergic inputs. Endocrinology. 2006 Jan; 147(1):359-66.

174 Landgren S, Wang MD, Bäckström T, Johansson S. Interaction between 3 alpha-hydroxy-5 alpha-pregnan-20-one and carbachol in the control of neuronal excitability in hippocampal slices of female rats in defined phases of the oestrus. Acta Physiol Scand. 1998 Jan;162(1):77-88.

175 Belelli D, Lambert JJ. Neurosteroids: endogenous regulators of the GABA(A) receptor. Nat Rev Neurosci. 2005 Jul;6(7):565-75.

176 Oakley AE, Clifton DK, Steiner RA. Kisspeptin signaling in the brain. Endocr Rev. 2009 Oct;30(6):713-43.

177 Jayasena CN, Nijher GM, Comninos AN, Abbara A, Januszewki A, Vaal ML, et al. The effects of kisspeptin-10 on reproductive hormone release show sexual dimorphism in humans. J Clin Endocrinol Metab. 2011 Dec; 96(12):E1963-72.

178 Dungan HM, Clifton DK, Steiner RA. Minireview: kisspeptin neurons as central processors in the regulation of gonadotropinreleasing hormone secretion. Endocrinology. 2006 Mar;147(3):1154-8.

179 Reddy DS, Jian K. The testosterone-derived neurosteroid androstanediol is a positive allosteric modulator of GABAA receptors. J Pharmacol Exp Ther. 2010 Sep;334(3): 1031-41.

180 Maguire J, Mody I. GABA(A)R plasticity during pregnancy: relevance to postpartum depression. Neuron. 2008 Jul;59(2):207-13. 
181 Agís-Balboa RC, Pinna G, Pibiri F, Kadriu B, Costa E, Guidotti A. Down-regulation of neurosteroid biosynthesis in corticolimbic circuits mediates social isolation-induced behavior in mice. Proc Natl Acad Sci USA. 2007 Nov; 104(47):18736-41.

182 Longone $\mathrm{P}$, Rupprecht R, Manieri GA, Bernardi G, Romeo E, Pasini A. The complex roles of neurosteroids in depression and anxiety disorders. Neurochem Int. 2008 Mar-Apr;52(4-5):596-601.

183 Earnheart JC, Schweizer C, Crestani F, Iwasato $\mathrm{T}$, Itohara $\mathrm{S}$, Mohler $\mathrm{H}$, et al. GABAergic control of adult hippocampal neurogenesis in relation to behavior indicative of trait anxiety and depression states. J Neurosci. 2007 Apr;27(14):3845-54.
184 Riecher-Rössler A. Sex and gender differences in mental disorders. Lancet Psychiatry. 2017 Jan;4(1):8-9.

185 Christiansen DM, Hansen M. Accounting for sex differences in PTSD: A multi-variable mediation model. Eur J Psychotraumatol. 2015 Jan;6(1):26068.

186 Pinares-Garcia P, Stratikopoulos M, Zagato A, Loke H, Lee J. Sex: a significant risk factor for neurodevelopmental and neurodegenerative disorders. Brain Sci. 2018 Aug;8(8):154.

187 Kuehner C. Why is depression more common among women than among men? Lancet Psychiatry. 2017 Feb;4(2):146-58.
188 Piran N. A feminist perspective on risk factor research and on the prevention of eating disorders. Eat Disord. 2010 May-Jun;18(3): 183-98.

$189 \mathrm{Li} \mathrm{SH}$, Graham BM. Why are women so vulnerable to anxiety, trauma-related and stress-related disorders? The potential role of sex hormones. Lancet Psychiatry. 2017 Jan;4(1):73-82.

190 Florent V, Baroncini M, Jissendi-Tchofo P, Lopes R, Vanhoutte M, Rasika S, et al. Hypothalamic structural and functional imbalances in anorexia nervosa. Neuroendocrinology. 2019, Epub ahead of print. 